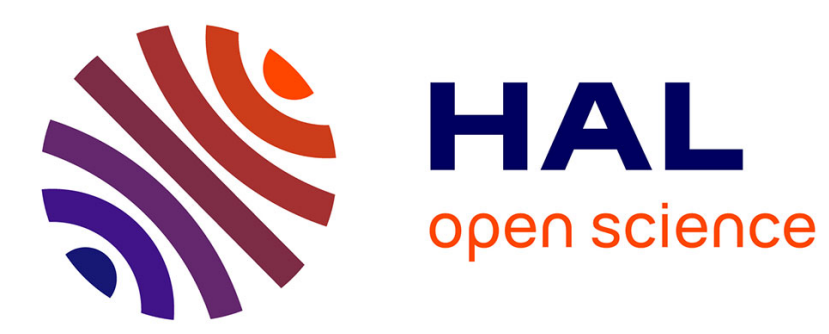

\title{
Adsorption of Atomic Hydrogen on Defect Sites of Graphite: Influence of Surface Reconstruction and Irradiation Damage
}

\author{
Christoph Lechner, Philippe Baranek, Holger Vach
}

\section{To cite this version:}

Christoph Lechner, Philippe Baranek, Holger Vach. Adsorption of Atomic Hydrogen on Defect Sites of Graphite: Influence of Surface Reconstruction and Irradiation Damage. Carbon, 2018, 10.1016/j.carbon.2017.09.095 . hal-02395184

\section{HAL Id: hal-02395184 \\ https://hal.science/hal-02395184}

Submitted on 5 Dec 2019

HAL is a multi-disciplinary open access archive for the deposit and dissemination of scientific research documents, whether they are published or not. The documents may come from teaching and research institutions in France or abroad, or from public or private research centers.
L'archive ouverte pluridisciplinaire HAL, est destinée au dépôt et à la diffusion de documents scientifiques de niveau recherche, publiés ou non, émanant des établissements d'enseignement et de recherche français ou étrangers, des laboratoires publics ou privés. 


\title{
Adsorption of Hydrogen on Defect Sites of Graphite: Influence of Surface Reconstruction and Irradiation Damage
}

\author{
Christoph Lechner ${ }^{\mathrm{a}}$, Philippe Baranek ${ }^{\mathrm{b}, *}$, Holger Vach $^{\mathrm{c}}$ \\ ${ }^{a}$ EDF RESD, Department Materials and Mechanics of Components (MMC), EDF Lab \\ Les Renardières, Avenue des Renardières, F-77818 Moret-sur-Loing Cedex, France \\ ${ }^{b}$ EDF REDD, Department Economic and Technical Analysis of Energy Systems (EFESE), \\ EDF Lab Chatou, 6 Quai Wattier, F-78400 Chatou Cedex, France \\ ${ }^{c}$ LPICM, CNRS, Ecole Polytechnique, Université Paris-Saclay, F-91128 Palaiseau, \\ France
}

\begin{abstract}
The influence of surface reconstruction and defects due to irradiation damage on the trapping of hydrogen in nuclear graphite has been investigated at the $a b$ initio level. Several models of defects and surfaces have been studied and compared with previously proposed traps, i.e. the zig zag edge of dislocation loops and reconstructed surfaces of graphite crystallites. The relative stabilities of hydrogen adsorption on the (100), (110), and (001) graphite basic planes have been evaluated for different amounts of hydrogen coverage and various types of reconstruction. The unreconstructed (100) surface adsorbs hydrogen the strongest. The (100) and (110) surface reconstructions result in a decreased stability for $\mathrm{H}$ adsorption compared to unrelaxed surfaces. Point defects caused by irradiation, such as mono- and divacancies, also trap hydrogen. We find that extended defects are weaker traps than monovacancies. This is true for surface defects as well as for bulk defects. The obtained results show that the existing hypothesis for trapping at dislocation loops has to be refined. Finally, an agreement with experiments is obtained for trapping on the reconstructed surfaces.
\end{abstract}

\footnotetext{
${ }^{*}$ Corresponding author.

Email address: philippe.baranek@edf.fr (Philippe Baranek)
} 


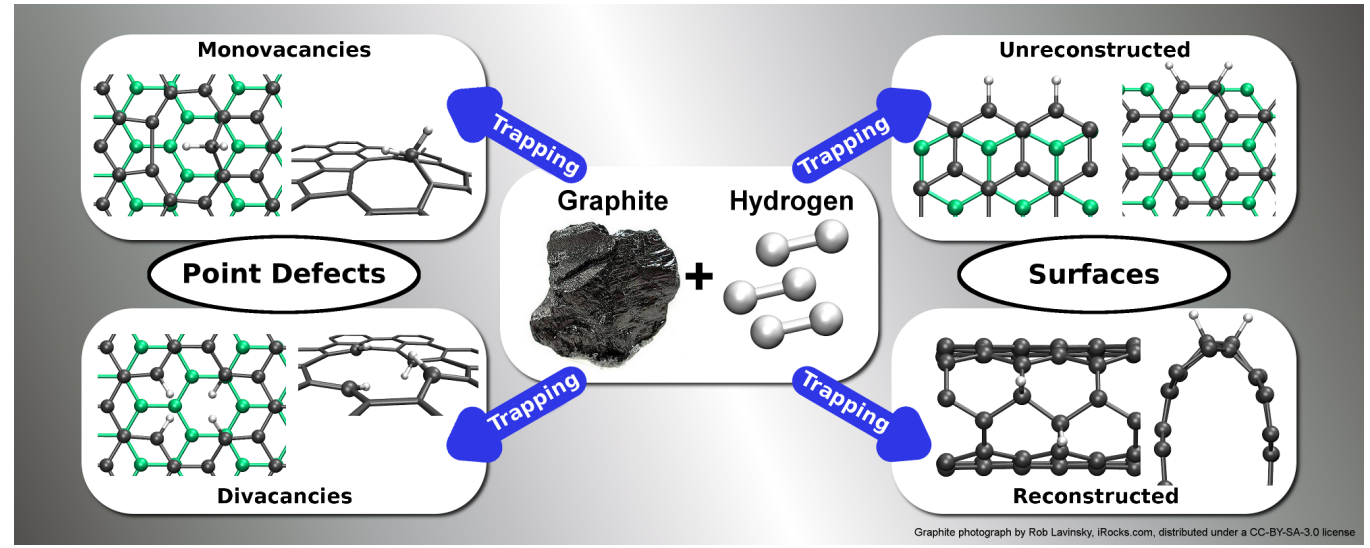

Figure for graphical abstract. 


\section{Introduction}

Significant interest in the properties of graphite has again arisen due to the upcoming dismantlement of several nuclear power plants of the UNGG (Uranium Naturel Graphite Gaz) type, which used nuclear graphite as a neutron moderator and deflector. For instance in France, the dismantlement will lead to 23000 tons of irradiated graphite. This type of graphite has a complex heterogeneous multi-scale structure which differs substantially from an ideal monocrystal of graphite [1-3]. However, in order to understand macroscopical data, theoretical studies at the nano- and microscopic scale are an important tool to explain underlying phenomena even though they rely on simpler model systems due to the limitations of computation power. They can help to get a detailed understanding of the behavior of the irradiated graphite in order to find an optimal solution for the permanent disposal.

The irradiated graphite is classified as nuclear waste containing radionuclides with low activity and long half-life. With a half-life of about 12.3 years, tritium is rather short lived, but contributes significantly to the initial activity of the waste. The high interest in its behavior in nuclear graphite is due to its potential risk of being released while the graphite waste is prepared and transported to the site for permanent disposal.

Atsumi and coworkers [4-12] have studied the diffusion and adsorption of hydrogen in graphite over a time span of more than two decades and successively refined and expanded their theory. Molecular hydrogen can freely diffuse through the open pores of graphite and reach the filler grains. The diffusion along the grain boundaries of the crystallites is assumed to consist of a sequence of dissociation and recombination reactions of hydrogen on the surfaces. Hydrogen adsorption in graphite follows Sievert's law for hydrogen pressures up to $10 \mathrm{kPa}[7]$ indicating that hydrogen is adsorbed in its atomic form. Diffusion measurements and thermal desorption spectroscopy could show that hydrogen is trapped in two different traps with different adsorption enthalpies. Kanashenko et al. [13] estimated the adsorption enthalpies theoretically. Following his nomenclature, trap 1 with an adsorption enthalpy of about $-4.4 \mathrm{eV}$ per $\mathrm{H}_{2}$ was attributed to zigzag edges of dislocation loops in the crystallite. Trap 2 was attributed to the (100) or zigzag edge surface of the crystallite with an adsorption enthalpy of about $-2.3 \mathrm{eV}$ per $\mathrm{H}_{2}$. Atsumi obtained $-4.4 \mathrm{eV}$ (trap 1) and $-2.6 \mathrm{eV}$ per $\mathrm{H}_{2}$ (trap 2) experimentally [11]. The difference is explained by the reconstruction of the (100) surface. Dislocation loops within the crystallite cannot reconstruct and thus saturation of the dangling bonds with hydrogen stabilizes the system to a higher extent compared to the surface. This part of the hypothesis relies until today on very crude calculations from the 1960's [14]. It is therefore important to re- 
view these assumptions with modern $a b$ initio methods to check the validity of this hypothesis.

Since graphite is also often considered as a potential material for hydrogen storage, a vast number of experimental and theoretical studies have already been published. For graphene, spin polarization of the edges [15-20] as well as the influence of edge reconstruction on hydrogen adsorption $[15,16,21]$ have been studied. However, a link to phenomena found in graphite has not yet been established. Considering graphite's literature, physisorption of atomic and molecular hydrogen on the (001) surface was studied on a theoretical level in Refs. [22-30]. However, these interactions are far too weak $(\approx 0.05 \mathrm{eV})$ compared to the experimentally measured adsorption energies and will not be considered in this paper. H can also chemisorb on this surface[22, 23, 26, 3133, 35-37]; for $\mathrm{H}_{2}$ desorption is thermodynamically preferred over adsorbed $\mathrm{H}$ dimers $[34,37]$. The chemisorption of these two species on the (100) or zigzag surface and (110) or armchair surface of graphite was shown by Yang et al. [38], Diño et al. [39, 40] and Sha et al. [41] studied the potential energy surface for $\mathrm{H}_{2}$ dissociation on these surfaces. In the graphite literature, it is not completely clear which surface binds hydrogen more strongly. Yang et al. [38] and Sha et al. [41] found a stronger adsorption on the (100) surface, whereas Diño et al. $[39,40]$ found a stronger adsorption on the (110) surface. Therefore, this problem needs to be treated with more accurate models to solve this ambiguity.

Since nuclear graphite was subject to irradiation during the operation of the nuclear power plants its crystalline filler grains differ quite substantially from an ideal HOPG monocrystal. The neutron irradiation is known to not only damage the surface of graphite. High-energy neutrons can create a cascade, which displaces carbon atoms hundreds of nm deep in the bulk [1]. Different types of point defects are known to occur in irradiated graphite. They include the Stone-Thrower-Wales defect, where a bond which is shared by two atoms is rotated by $90^{\circ}$. The properties of the defects themselves as well as the chemisorption of atomic hydrogen have already been studied in detail [42-45]. When the kinetic energy of a particle is high enough (> $30 \mathrm{eV}$ ), a Frenkel-pair is created (see Ref. [1] and references therein). This interstitial-vacancy pair as well as other single interstitials have already been investigated [42, 46, 47]. In general, interstitials are found to be more mobile than vacancies [48]. This leads to a higher concentration of monovacancies compared to single interstitials [1]; therefore, we will focus on them. Since monovacancies have dangling bonds, they may serve as additional traps for hydrogen. Several authors have shown that $\mathrm{H}$ is strongly chemisorbed at monovacancies [49-51]. However, diffusion of vacancies is possible as well either by thermal excitation or irradiation. Theoretical predictions for the 
migration barrier of a single vacancy lie in the range of $1.3-1.6 \mathrm{eV}[48,52,53]$ and are in agreement with the activation energy of $1.8 \mathrm{eV}$ obtained from Raman measurements [54]. The height of this barrier allows for an accumulation of vacancies to occur which can create divacancies or even extended vacancy lines (see Ref. [1] and references therein). As pointed out in Ref. [48] and also shown by molecular dynamics simulations [55], coalescence of two monovacancies to form a divacancy has a barrier of $2.2 \mathrm{eV}$. UNGG reactors operated with high temperature differences $\left(150-450{ }^{\circ} \mathrm{C}\right)$, however as shown in Ref. [56] monovacancies are systematically annealed from $200{ }^{\circ} \mathrm{C}$ onwards. Thus, we need to consider both monovacancies as well as extended vacancies to describe the possible interactions between hydrogen and defects due to irradiation. A 5-8-5 divacancy still allows for a reasonable model system size, but already possesses important features of vacancy lines (such as a reconstruction with no dangling bonds remaining). It is therefore important to study the effect of extended defects on the adsorption properties.

In this paper the (001), (100), and (110) surfaces as well as monovacancies and the 5-8-5 divacancy in the (001) surface will be used as model systems to get an understanding of the chemisorption of hydrogen at surfaces and defect sites. This work focuses also on an effect that has never been considered at the $a b$ initio level to our knowledge: the restructuring of the surface and its influence on the adsorption of hydrogen. While this effect was acknowledged in Kanashenko's paper and later on in Atsumi's work, their models are based on very crude calculations from the 1960's [14]. Surface reconstruction was observed by conducting TEM and HRTEM measurements (see Ref. [57] and references therein) and also studied theoretically for graphene bilayers [58] and nanoribbons $[15,18,21,59]$. Different types of reconstruction were found; in this work two different kinds have been treated: In-plane reconstructions of edge carbon atoms and bond formation between edge carbon atoms of two adjacent graphene planes yielding arch-shaped edges.

Finally, by approximating Kanashenko's traps [13] with our model systems we can then compare the existing hypothesis with our results.

The paper is organized as follows: in the next section we explain the methods used for this study. After that we present and discuss the results of this work. Three different traps for hydrogen are investigated: unreconstructed surfaces, reconstructed surfaces, and mono- and divacancies. We provide evidence that the existing hypothesis is outdated for the first trap and find agreement for the second trap. We show that mono- and divacancies also serve as strong traps. Finally, we conclude on our findings. 


\section{Methodology}

The calculations were performed with the periodic CRYSTAL09 [60, 61] code in which the most recent dispersion correction D3 of Grimme [62] was implemented [63]. The PBE (for Perdew-Burke-Ernzerhof exchangecorrelation) functional [64] combined with the D3 dispersion correction [62] as parametrized in reference [63] was used to describe the properties of graphite. Regarding the parameterization of the D3 correction the zero damping was used: the $s_{6}, s_{8}, s_{r, 6}$ and $s_{r, 8}$ scaling parameters were set to $1.0,0.410,1.217$ and 1.0, respectively. The cutoff values for the dispersion interaction and coordination numbers were 50 and $20 \AA$, respectively. Three-body terms were not included. This parametrization coupled with a GTF basis set appears to be a good compromise for an accurate description of the structural, electronic, dynamic, and mechanical properties of graphite as shown in our recently published work [63] : The obtained lattice parameters ( $a$ and $c$ ) are $2.463 \AA, 6.671 \AA$, and the BSSE corrected cohesive and interlayer interaction energies are $7.88 \mathrm{eV} /$ atom and $43 \mathrm{meV} /$ atom compared to the experimental data $2.459 \AA[65], 6.672 \AA[65], 7.36 \mathrm{eV} /$ atom [66] and $35-52 \mathrm{meV} /$ atom [67, 68], respectively.

For the Gaussian basis sets carbon and hydrogen were described by an all-electron basis set. The $\mathrm{C}$ basis set $[69,70]$ is the same as reported in our previous work on graphite [63], a $6 s-211 s p-1 d^{*}$ basis (a contraction of 6,2 , 1,1 , and 1 Gaussian- type functions for the $1 s, 2 s p, 3 s p, 4 s p$, and $d$ shells, respectively). The $\mathrm{H}$ basis set [71] is a contraction of $-511 s-1 p^{*}-$ GTFs and is fully described in the Supporting Information.

In this paper, the two dimensional (2-D) slab model is used to study the surface properties: the surface consists of a slab formed by a sufficient number of atomic layers parallel to a given plane. It is limited by two surface planes and has 2-D periodic boundaries conditions; thus in this framework the system is two dimensional and isolated. To treat the defective crystals and adsorbed surfaces a supercell approach has been adopted. The sizes of the used supercells were $5 \times 5 \times 2$ for bulk HOPG, a $5 \times 5$ supercell of a 4 -layer slab for the (001) surface, a $3 \times 2$ supercell of a 24-layer slab for the (100) surface, and a $2 \times 2$ supercell of a 14-layer slab for the (110) surface. The number of carbon atoms for these different models ranges from 192 to 224 atoms. For the in-plane reconstructed surfaces, we used a $4 \times 2$ supercell of a 24-layer slab for the (100) surface, and a $2 \times 2$ supercell of a 14-layer slab for the (110) surface; for arch-shaped reconstructions a $3 \times 1$ supercell of a 66 layer slab for the (100) surface and a $2 \times 1$ supercell of a 36 -layer slab for the (110) surface. The various cells are sufficiently large (224-288 carbon atoms) to allow full relaxation of the reconstructed surfaces. Hydrogen was always 
adsorbed only on one side of the slab. Adsorption of multiple hydrogen atoms is done successively, thus the optimized structure for $(n-1)$ hydrogen atoms is the starting point for adsorption of $n$ hydrogen atoms. Only the most stable position for the additional hydrogen is reported.

The methods for the calculation of surface energies $\mathrm{E}_{\text {surf }}$ and formation energies $\mathrm{E}_{\text {form }}$ of the studied surfaces and vacancies are described in the Supporting Information. The adsorption energy $\mathrm{E}_{a d}$ is defined as the energy difference between the energy of the graphite-adsorbate structure and the sum of energies of the graphite structure and a hydrogen in its reference state:

$$
E_{a d}=\frac{E_{0}-E_{G}^{o p t}-n \cdot E_{H}}{n}
$$

where $E_{0}$ is the total energy of the optimized system, $E_{G}^{o p t}$ the energy of the optimized surface without adsorbates, $E_{H}$ the energy of the isolated hydrogen and $n$ the number of adsorbed $\mathrm{H}$-atoms. All $\mathrm{E}_{a d}$ are calculated with respect to atomic hydrogen. In some cases we also consider $\mathrm{E}_{a d}$ with respect to $\mathrm{H}_{2}$ for the purpose of discussion and linking to experimental results, here we use a binding energy of $-4.5 \mathrm{eV}$ for the $\mathrm{H}_{2}$ molecule.

Since Gaussian-type basis sets suffer from the basis set superposition error (BSSE) we corrected our adsorption energy for this error by using a modified version of the counterpoise method of Boys and Bernardi [72]. This is described in detail in the Supporting Information. To quantify the influence of surface restructuring we define the deformation energy $\mathrm{E}_{\text {def }}$ as the energy difference of the optimized pristine surface and the optimized surface deformed due to the adsorption.

All computational parameters necessary to reproduce our results are given in the Supporting Information. All determined total energies, formation energies of the surfaces and adsorption energies are given at the athermal limit $(\mathrm{T}=0 \mathrm{~K})$, thus thermal contributions are not considered.

\section{Results and Discussion}

\subsection{Surfaces}

The surface energies of the (001), (100), and (110) surface were determined and are given in Table 1. The (001) surface has the smallest surface energy since no $\mathrm{C}-\mathrm{C}$ bonds are broken in order to create it. Compared to the (001) surface, the (100) and (110) surface energies are higher by a factor of 30 - 40, the (100) surface has the highest one. The obtained relative stability of the (100) and (110) surface is due to atomic relaxation processes at the surface, which are much more pronounced at the (110) surface. Both relaxed surfaces are shown in Figure 1 together with the periodic boxes. When the 
(110) surface is allowed to relax it forms stronger bonds between pairs of edge carbon atoms. This decreases the C1-C2 (C3-C4) bond length from $1.42 \AA$ to about $1.23 \AA$ (see Figure 1 for the description of atoms), which is an indication that the bond is almost a triple bond (the bond length in ethyne is $1.20 \AA$ ). The ground state of the (110) surface shows no spin localization at the edge carbon atoms and is non-magnetic.

Table 1: Surface energies $\left(\mathrm{E}_{\text {surf }}\right.$ in $\left.\mathrm{J} / \mathrm{m}^{2}\right)$ of the three studied surfaces.

\begin{tabular}{cc}
\hline System & $\mathrm{E}_{\text {surf }}$ \\
\hline$(001)$ & 0.19 \\
$(100)$ & 6.15 \\
$(110)$ & 5.29 \\
\hline
\end{tabular}

The (100) surface hardly changes when it is optimized. The edge carbon atoms move slightly inward by $0.03 \AA$, the angle between the edge carbon and its two carbon neighbors, $\measuredangle \mathrm{C} 4-\mathrm{C} 1-\mathrm{C} 5$, increases to 126 degrees. However, a strong spin localization at the edge carbon atoms is observed. This is in line with the findings of several authors $[17,18,76]$. Each edge carbon has a magnetic moment of roughly $1.3 \mu_{B}$.

Atomic hydrogen is rather reactive with respect to graphite. It is known to chemisorb on the pristine (001) surface by forming a weak covalent bond with a carbon atom of the surface. The two possible adsorption sites are shown in Figure 2. The adsorption is linked to a lifting of this carbon atom out of the graphene plane by about $0.49 \AA$ (puckering) due to the increase of its $s p^{3}$ character (see Figure 2). $\mathrm{E}_{a d}$ for both positions are very similar: $0.68 \mathrm{eV}$ and $-0.73 \mathrm{eV}$ for position 1 and 2, respectively. This weak adsorption energy is also reflected in a rather long $\mathrm{C}-\mathrm{H}$ bond of $1.13 \AA$ (for $\mathrm{CH}_{4}$ it is 1.09 $\AA)$. Our results are in good agreement with literature values for graphene, which range from -0.87 to $-0.67 \mathrm{eV}[23,49,77-83]$. For comparison we studied the insertion of hydrogen in bulk graphite. As for the surface, hydrogen forms a weak covalent bond with the graphite matrix. The insertion energies are almost equal to the adsorption energies for the (001) surface: $-0.63 \mathrm{eV}$ and $-0.76 \mathrm{eV}$ for position 1 and 2, respectively. Position 2 is slightly preferred since the hydrogen is centered exactly above the center of a six-membered ring.

Contrary to the (001) surface, both the (100) and (110) surfaces have dangling bonds, which become saturated when hydrogen is adsorbed. The adsorption of $\mathrm{H}_{2}$ on the (100) and (110) surface of HOPG was studied by Diño et al. $[39,40]$ However, since they explored the potential energy surface of this process they did not optimize the surface (all C-C bond lengths are $1.42 \AA$ ). 

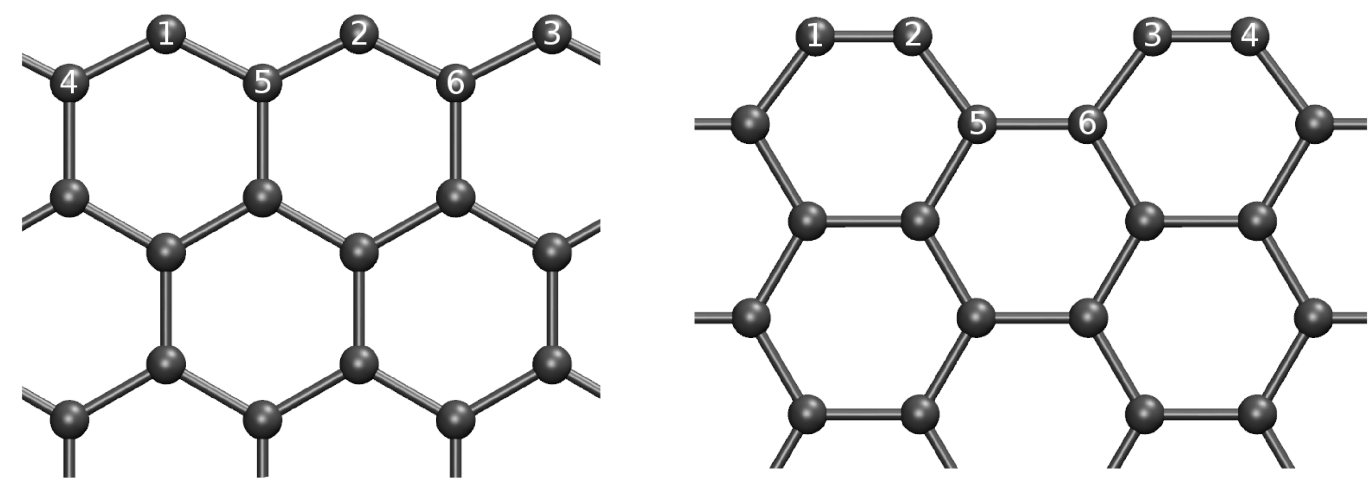

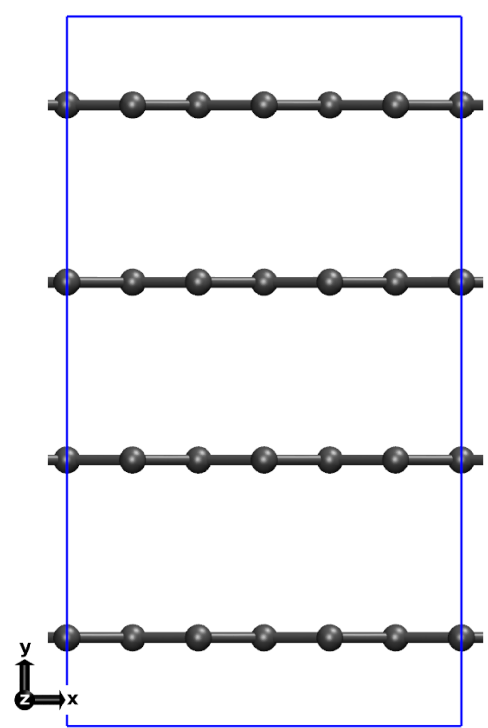

(a)

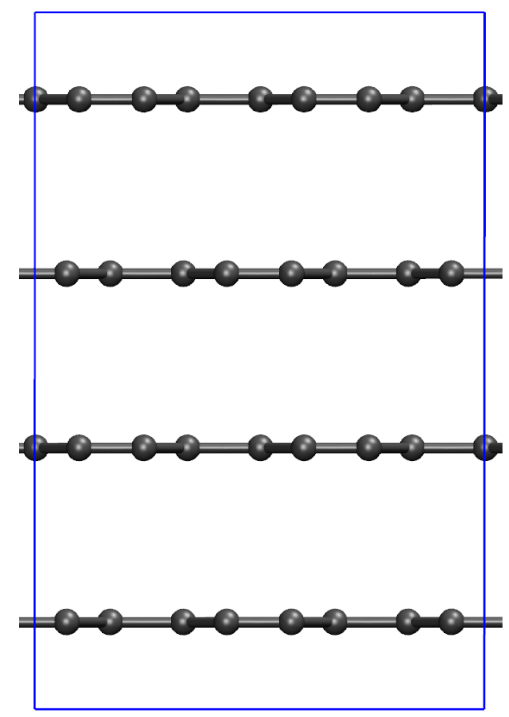

(b)

Figure 1: Optimized structures and top view of the (a) (100) and (b) (110) surfaces.

This leads to a broad range of their $\mathrm{E}_{a d}$ : from -5.0 to $-4.0 \mathrm{eV}$ per $\mathrm{H}_{2}$ for (110) and from -4.7 to $-3.2 \mathrm{eV}$ per $\mathrm{H}_{2}$ for (100). All the other available literature values are reported as $\mathrm{E}_{a d}$ per $\mathrm{H}$ atom. The $\mathrm{H}_{2}$ bond energy is about $-4.5 \mathrm{eV}$ (at the PBE level); this yields $\mathrm{E}_{a d}$ from -4.8 to -4.3 and from -4.6 to $-3.9 \mathrm{eV}$ per $\mathrm{H}$ for the (110) and (100) surface, respectively. The calculated $\mathrm{E}_{a d}$ for adsorption of two $\mathrm{H}$ on the (110) and (100) surface of Yang et al. [38] are -3.7 and $-3.9 \mathrm{eV}$ per $\mathrm{H}$, respectively. Sha et al. [41] obtained an $\mathrm{E}_{a d}$ of -5.0 $\mathrm{eV}$ for $\mathrm{H}$ on the (100) surface. Thus, in the literature it is not completely clear which surface binds hydrogen more strongly. 


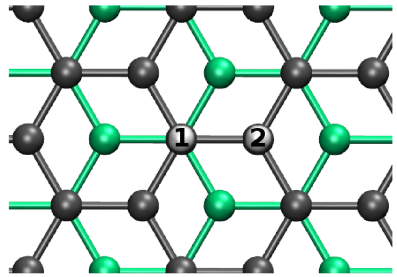

(a)

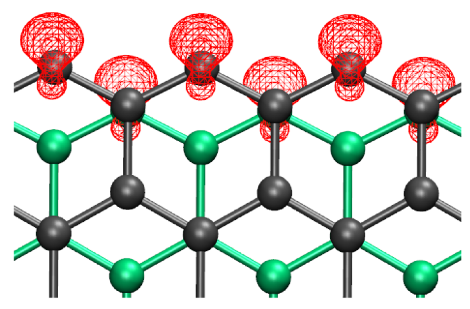

(d)

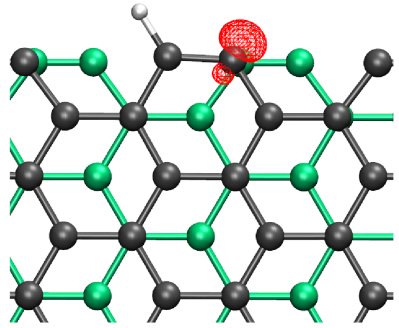

(g)

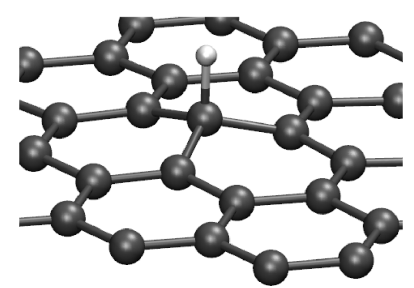

(b)

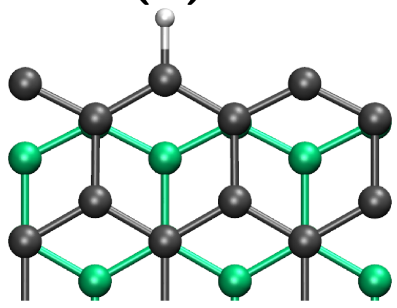

(e)

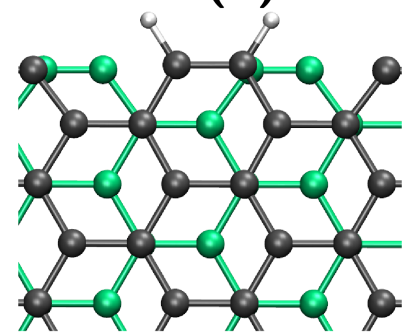

(h)

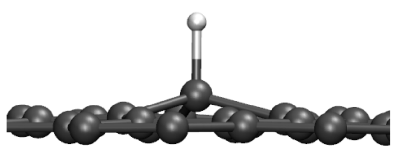

(c)

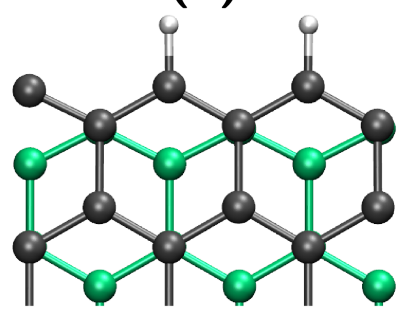

(f)

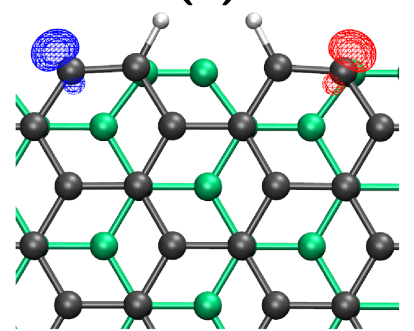

(i)

Figure 2: Optimized structures obtained for different cases of hydrogen adsorption on the (001), (100) and (110) surfaces. (a) Top view of the two adsorption sites for hydrogen on the (001) surface. (b) Top and (c) side views of the chemisorption of hydrogen on the (001) surface. (d) Spin density plot for the (100) surface. Each edge carbon has a localized spin. (100) surface with (e) one and (f) two chemisorbed hydrogen atoms. Spin density plots are omitted for better visibility, but all unsaturated edge carbon atoms have a localized spin. (g) Spin density plot for the (110) surface with one chemisorbed hydrogen. (h) and (i) show the (110) surface with two chemisorbed hydrogen atoms for the (h) $110-2 \mathrm{H}-\mathrm{a}$ and (i) 110-2H-b configurations, respectively. Isovalues for spin density plots are 0.05 (red) and -0.05 (blue) e/bohr ${ }^{3}$. 
Table 2: Hydrogen adsorption ( $\mathrm{E}_{a d}$ in $\mathrm{eV} / \mathrm{H}$ atom) and deformation ( $\mathrm{E}_{\text {def }}$ in $\mathrm{eV}$ ) energies on the different graphite surfaces with respect to the number of adsorbed H. $(100)_{\text {rec }} /(110)_{\text {rec }}$ denote the in-plane reconstructions, $(100)_{\text {arch }} /(110)_{\text {arch }}$ and $(100)_{\text {arch }}^{\text {bilayer }} /(110)_{\text {arch }}^{\text {bilayer }}$ denote the slab and bilayer models of the arch-shaped reconstruction, respectively. $\operatorname{Vac}(\alpha, \beta)$ and Divac denote the (001) surface with mono- and 5-85-divacancies, respectively. The different explored configurations are: a) on the same graphene plane, b) on two different graphene planes, c) two $\mathrm{H}$ per edge carbon, d) 110$2 \mathrm{H}-\mathrm{a}$, e) $110-2 \mathrm{H}-\mathrm{b}$, f) $\left.\left.100_{r e c}-2 \mathrm{H}-\mathrm{a} / 110_{r e c}-2 \mathrm{H}-\mathrm{a}, \mathrm{g}\right) 100_{r e c}-2 \mathrm{H}-\mathrm{b} / 110_{\text {rec }}-2 \mathrm{H}-\mathrm{b}, \mathrm{h}\right)$ ortho, i) meta and j) para (see Figure 2); k) correspond to the vacancies on the (001) surfaces (in parentheses, the adsorption energies of $\mathrm{H}$ with the same defects in bulk graphite are given for comparison).

\begin{tabular}{|c|c|c|c|c|}
\hline System & $\mathrm{Nb}$. of $\mathrm{H}$ & Conf. & $\mathrm{E}_{a d}$ & $\mathrm{E}_{\text {def }}$ \\
\hline \multirow{6}{*}{ (100) } & 1 & - & -4.99 & 0.05 \\
\hline & & $\mathrm{a}$ & -4.99 & 0.10 \\
\hline & 2 & $\mathrm{~b}$ & -5.00 & 0.11 \\
\hline & & $\mathrm{c}$ & -3.89 & 0.60 \\
\hline & 3 & - & -4.98 & 0.13 \\
\hline & 4 & $\mathrm{C}$ & -3.63 & 1.12 \\
\hline \multirow{8}{*}{$(110)$} & 1 & - & -3.73 & 0.54 \\
\hline & & d & -4.37 & 0.72 \\
\hline & 7 & $\mathrm{e}$ & -3.64 & 1.02 \\
\hline & 2 & b & -3.73 & 1.08 \\
\hline & & $\mathrm{c}$ & -2.68 & 1.59 \\
\hline & 3 & - & -4.08 & 1.13 \\
\hline & & $\mathrm{a}$ & -4.29 & 1.25 \\
\hline & 4 & $\mathrm{c}$ & -3.40 & 2.54 \\
\hline \multirow{3}{*}{$(100)_{r e c}$} & 1 & - & -2.80 & 0.93 \\
\hline & & $\mathrm{f}$ & -3.80 & 1.35 \\
\hline & 2 & $g$ & -2.81 & 1.78 \\
\hline \multirow{3}{*}{$(110)_{r e c}$} & 1 & - & -2.63 & 0.79 \\
\hline & & $\mathrm{f}$ & -3.68 & 1.27 \\
\hline & 2 & g & -2.62 & 1.55 \\
\hline \multirow{3}{*}{$(100)_{a r c h}$} & 1 & - & -2.19 & 0.77 \\
\hline & 2 & $\mathrm{~h}$ & -2.86 & 1.52 \\
\hline & & $\mathrm{i}$ & -2.35 & 1.61 \\
\hline \multirow{4}{*}{$(110)_{a r c h}$} & 1 & - & -2.39 & 0.61 \\
\hline & & $\mathrm{h}$ & -2.56 & 1.20 \\
\hline & 2 & $\mathrm{i}$ & -2.42 & 1.40 \\
\hline & & $\mathrm{j}$ & -2.64 & 1.24 \\
\hline \multirow{4}{*}{$(100)_{\text {arch }}^{\text {bilayer }}$} & 1 & - & -1.24 & 1.01 \\
\hline & & $\mathrm{h}$ & -2.02 & 2.34 \\
\hline & 2 & $\mathrm{i}$ & -1.37 & 2.46 \\
\hline & & $\mathrm{j}$ & -1.88 & 2.08 \\
\hline \multirow{4}{*}{$(110)_{\text {arch }}^{\text {bilayer }}$} & 1 & - & -1.28 & 1.00 \\
\hline & & $\mathrm{h}$ & -2.22 & 2.54 \\
\hline & 2 & $\mathrm{i}$ & -1.37 & 2.26 \\
\hline & & $\mathrm{j}$ & -1.94 & 1.98 \\
\hline \multirow{2}{*}{$\operatorname{Vac}(\alpha)$} & 1 & $\mathrm{k}$ & $-4.17(-4.13)$ & $0.25(0.26)$ \\
\hline & 2 & $\mathrm{k}$ & $-3.34(-3.39)$ & $1.16(0.39)$ \\
\hline \multirow{2}{*}{$\operatorname{Vac}(\beta)$} & 1 & k 11 & $-4.15(-4.15)$ & $0.28(0.22)$ \\
\hline & 2 & $\mathrm{k}$ & $-3.31(-3.35)$ & $1.19(0.35)$ \\
\hline \multirow{4}{*}{ Divac } & 1 & $\mathrm{k}$ & $-2.27(-2.21)$ & $0.77(0.69)$ \\
\hline & 2 & $\mathrm{k}$ & $-3.28(-3.30)$ & $2.31(2.24)$ \\
\hline & 3 & $\mathrm{k}$ & -3.25 & 3.68 \\
\hline & 4 & $\mathrm{k}$ & -3.55 & 3.98 \\
\hline
\end{tabular}


Adsorption on the (100) surface is a much more stabilizing process $\left(\mathrm{E}_{a d}\right.$ differs by a factor of 7) compared to the adsorption on the (001) surface (see Table 2). The adsorption of $\mathrm{H}$ on the (100) surface hardly perturbs the surface structure: $\mathrm{E}_{\text {def }}$ of the lattice is only $0.05 \mathrm{eV}$ (see Table 2).

The ground state of the (100) surface is the high spin state. For each adsorbed hydrogen the total magnetic moment decreases by one. The magnetic moment of the neighboring edge carbon atoms shows no significant change.

When a second hydrogen adsorbs on the same graphene plane of this surface $\mathrm{E}_{a d}$ doubles. This shows that there is almost no interaction between neighboring hydrogen atoms (they are $2.5 \AA$ apart) and that the adsorption of $\mathrm{H}$ on the (100) surface is a highly local process.

For three adsorbed hydrogen atoms which corresponds to a fully covered plane in our model, the angle $\measuredangle \mathrm{C} 4$-C1-C5 decreases to 122 degrees and approaches the ideal value of 120 degrees for a $s p^{2}$ carbon as in benzene.

$\mathrm{E}_{\text {def }}$ for 1 to 3 adsorbed hydrogen atoms never exceeds $0.13 \mathrm{eV}$, which is almost negligible. This shows that the graphite matrix does not change significantly upon hydrogen adsorption since the edge carbon atoms are almost perfectly oriented to bind an approaching hydrogen.

Furthermore, when two hydrogen atoms bind to two edge carbon atoms which belong to two different planes, there is hardly any difference between the two adsorption energies (the energy difference $\Delta \mathrm{E}$ is $0.01 \mathrm{eV}$ ). This shows as well that the hydrogen atoms barely interact with each other. The structure of Diño et al. [39] which yields an $\mathrm{E}_{a d}$ of $-4.6 \mathrm{eV}$ resembles this structure. Their result agrees rather well with our $\mathrm{E}_{a d}$ of $-5.0 \mathrm{eV}$ (full relaxation of Diño's structures might yield even better agreement). We find excellent agreement with the result of $-5.0 \mathrm{eV}$ reported by Sha et al. [41].

Hydrogen adsorption on the (110) surface is also a more stabilizing process $\left(\mathrm{E}_{a d}\right.$ differs by a factor of 5) compared to the adsorption on the (001) surface. When atomic hydrogen adsorbs on the (110) surface and forms a bond with $\mathrm{C} 1$ the local deformation of the graphite lattice is more pronounced. The ground state has a magnetic moment of $1.0 \mu_{B}$; the unpaired electron is strongly localized on the neighboring carbon atom $\mathrm{C} 2$ as shown in the spin density plot in Figure 2. This adsorption leads to a weakening of the C1-C2 bond, which can be seen by the $\mathrm{C} 1-\mathrm{C} 2$ bond length increase to about 1.34 $\AA$, and the $\mathrm{C} 1-\mathrm{C} 2$-C5 angle decrease by almost 10 degrees. The weakening of the C1-C2 bond as well as the local deformation of the surface are major factors for this $\mathrm{E}_{a d}$ being higher than on the (100) surface, as shown in Table 2. $\mathrm{E}_{\text {def }}$ is 10 times higher for the (110) surface $(0.54 \mathrm{eV})$, which accounts for $40 \%$ of the difference in adsorption energies. The other part is due to the spin localization.

There are two ways to add a second hydrogen adjacent to the first one on 
the same graphene plane: either on the same six-membered ring $(\mathrm{C} 2)$ or on the neighboring six-membered ring (C3). We call these two configurations 110-2H-a and 110-2H-b, respectively. They are shown in Figure 2. For these structures we find major differences. The configuration $110-2 \mathrm{H}-\mathrm{a}$ is largely favored; $\mathrm{E}_{a d}$ per $\mathrm{H}$ atom differs by $0.8 \mathrm{eV}$ with respect to $110-2 \mathrm{H}-$ b. This is due to the fact that the saturation of both adjacent edge carbon atoms recreates a system of conjugated bonds. The $\mathrm{C} 1-\mathrm{C} 2$ bond length is $1.37 \AA$, the angle $\measuredangle \mathrm{C} 2-\mathrm{C} 1-\mathrm{C} 5$ (and its symmetric counterpart) for the edge carbon atoms are 120.6 degrees. This is not the case for $110-2 \mathrm{H}-\mathrm{b}$, in fact its formation creates another local deformation of a six-membered ring. $\mathrm{E}_{\text {def }}$ is $0.25 \mathrm{eV}$ higher with respect to $110-2 \mathrm{H}-\mathrm{a}$. The ground state of $110-2 \mathrm{H}-\mathrm{b}$ is antiferromagnetic, but the magnetic state with $2.0 \mu_{B}$ lies only $0.06 \mathrm{eV}$ higher in energy. Diño et al.[40] found the same trend for the difference of the adsorption energies $(0.5 \mathrm{eV} / \mathrm{H})$ of the two configurations, but their adsorption energies are underestimated by $0.4 \mathrm{eV}$ for $110-2 \mathrm{H}-\mathrm{a}$ and $0.7 \mathrm{eV}$ for $110-2 \mathrm{H}-\mathrm{b}$. However, since their graphite surface is not fully relaxed the contribution of lattice deformation energy is excluded, which decreases adsorption energies.

For the adsorption of a third hydrogen the only possibility is to bind to a carbon with an unsaturated edge carbon neighbor, thus the same trends as for one adsorbed $\mathrm{H}$ are observed. For a fourth adsorbed hydrogen the trend of $110-2 \mathrm{H}-\mathrm{a}$ is reproduced.

The same trend as for configuration 110-2H-b is found when the two hydrogen atoms adsorb on two carbon atoms which belong to different graphene planes. The slightly lower $\mathrm{E}_{a d}(\Delta \mathrm{E}=0.09 \mathrm{eV})$ is due to less steric repulsion between the hydrogen atoms for this structure.

To summarize, for the (110) surface the adsorption processes are no longer independent. Formation of 110-2H-a like structures is favored over other structures; this configuration is more stable by at least $0.6 \mathrm{eV}$ per $\mathrm{H}$ atom compared to all the other.

For both surfaces, (100) and (110), it is possible that two hydrogen atoms adsorb on one edge carbon. This leads to a $s p^{3}$ hybridization of the edge carbon. Adsorption on the (100) surface is more stable, $\mathrm{E}_{a d}$ per $\mathrm{H}$ atom differs by $1.2 \mathrm{eV}$ per $\mathrm{H}$ atom. This trend agrees qualitatively with the results of Yang et al.[38], but their $\mathrm{E}_{a d}$ are overestimated by 1.1 and $0.7 \mathrm{eV}$ per $\mathrm{H}$ atom for the (100) and (110) surface, respectively. The angle $\mathrm{H}-\mathrm{C} 1-\mathrm{H}$ takes 106.5 degrees for the (100) and 105.2 degrees for the (110) surface, thus showing the increased $s p^{3}$ character of the edge carbon. Both hydrogen atoms are above the interlayer space (see Supporting Information). Since these structures are far less stable $(\Delta \mathrm{E}$ is 1.07 and $1.69 \mathrm{eV}$ per $\mathrm{H}$ atom for the (100) and (110) surfaces, respectively) we only report $\mathrm{E}_{a d}$ for adsorption on one and two carbon atoms in Table 2. 
When adsorption of hydrogen on the three surfaces is compared, the trend of stability is $(100)>(110)>(001)$, thus the (100) surface forms the most stable bonds. Considering the surface energies of these three surfaces, it can be noticed that these two properties are directly related: A higher surface energy leads to a more stable structure for hydrogen adsorption.

As written in the introduction trap 1 in nuclear graphite is supposed to be the zigzag edges of dislocation loops. Following Kanashenko's hypothesis we assume our (100) surface model to be equivalent to these edges. We get an adsorption energy of about $-5.5 \mathrm{eV} / \mathrm{H}_{2}$ compared to Kanashenko's -4.4 $\mathrm{eV} / \mathrm{H}_{2}$, which is a quite large difference. However, Kanashenko obtained his result by assuming a $\mathrm{C}-\mathrm{H}$ bond energy of $-4.45 \mathrm{eV}$ (taken from the bond energy of benzene) and a $\mathrm{H}-\mathrm{H}$ bond energy of $-4.5 \mathrm{eV}$. While the $\mathrm{H}-\mathrm{H}$ bond energy is correct the $\mathrm{C}-\mathrm{H}$ bond energy for benzene was shown to be -4.86 $\mathrm{eV}$ [84]. This would result in an estimate of $-5.2 \mathrm{eV}$, which is much closer to our result. The most recent experimental adsorption energy is reported as $-4.6 \mathrm{eV}$ [11]. Thus, both Kanashenko's estimate and our result for the zigzag edges underestimate this experimental result (by $0.6 \mathrm{eV}$ and $0.9 \mathrm{eV}$, respectively). For prismatic edge dislocations Suarez-Martinez et al. [85] showed that the zigzag edge carbon atoms actually form two interlayer single bonds and become fully saturated. However, the armchair edge forms no interlayer bonds and is very similar to our (110) model surface. This suggests that only the armchair edges are available for hydrogen adsorption without breaking of C-C bonds. $\mathrm{E}_{a d}$ for configurations $110-2 \mathrm{H}-\mathrm{a}$ and $110-2 \mathrm{H}-\mathrm{b}$ of our (110) surface model are $-4.2 \mathrm{eV} / \mathrm{H}_{2}$ and $-2.8 \mathrm{eV} / \mathrm{H}_{2}$, respectively. The more stable configuration 110-2H-a overestimates the experimental results only by $0.4 \mathrm{eV}$. Thus the measured value lies between the theoretical results of our two model systems. The differences could be due to the difficulty to compare experimental data measured at elevated temperatures $\left(>750{ }^{\circ} \mathrm{C}\right)$ with theoretical $a b$ initio results of well defined structures at $0 \mathrm{~K}$. Our results clearly show that Kanashenko's conclusion for trap 1 in nuclear graphite needs a revision. First, his estimation needs to be corrected and second, both zigzag and armchair edges should be considered as traps.

In the next section we will focus on the change of adsorption properties of hydrogen when the surfaces (100) and (110) are allowed to reconstruct.

\subsection{Reconstructed Surfaces}

Surface reconstructions of the (100) and (110) surface can be divided into two groups. The first group consists of reconstructions where edge carbon atoms of one graphene plane can regroup to create patterns which differ from the zigzag or armchair edges. For graphene nanoribbons, several reconstructed surfaces as well as their interaction with hydrogen were studied on 
a theoretical level $[15,18,21]$. For our study we chose the two reconstructions which yield weaker adsorption energies than for the unreconstructed armchair edge since this makes them possible candidates for the second trap according to Kanashenko's and Atsumi's hypothesis. These in-plane reconstructions are shown in Figure 3. The first reconstruction stems from the (100) surface by rotating a C-C bond by $90^{\circ}$ similar to the Stone-ThrowerWales defect (C5-C6 in Figure 3). It results in a sequence of heptagons and pentagons. The second one stems from the (110) surface and yields a sequence of two heptagons and one hexagon by rotating a $\mathrm{C}-\mathrm{C}$ bond which connects two armchair hexagons by $90^{\circ}$ (C5-C6 in Figure 3).

The second group consists of reconstructions where dangling bonds of the (100) and (110) surface are saturated by forming bonds between edge carbon atoms of two adjacent graphene planes, called arch-shaped edges. This effect was observed by conducting TEM and HRTEM measurements (see Ref.[57] and references therein). As shown in Figure 3 we restrict ourselves to two limiting cases for the arch-shaped edges with respect to the curvature of the arch: The first case is the surface reconstruction of a graphene bilayer which is only periodic in one dimension and allows full relaxation orthogonal to the graphene plane (called bilayer model). This reconstruction results in a low curvature. The second case is the surface reconstruction of slab models with two graphene planes per supercell which is periodic in 2 dimensions and limits the relaxation to give a surface with a high curvature (called slab model). The difference in periodicity of these two models as well as a schematic description of the reconstruction process are shown in the Supporting Information. A model similar to our slab model was already used to study the chemistry of sulfur in graphite [87].

The formation energies of the different reconstructed surfaces are given in Table 3. The in-plane reconstruction is only exothermic for the (100) surface which is in line with Ref. [21]. For all arch-shaped edges this process leads to more stable structures since no dangling bonds remain. We also find that reconstruction of the (100) surface is systematically more stabilizing whatever the reconstruction type. This is coherent with the higher surface energy.

For both in-plane reconstructions studied the edge carbon atoms which are available for hydrogen adsorption belong to heptagons. The local structure is similar to the (110) surface, C1-C2 bond lengths for both systems decrease to $1.24 \AA$ (see Figure 3 for the description of atoms). The optimized structures for adsorption of one and two $\mathrm{H}$ atoms on the two studied reconstructed surfaces are shown in Figure 4. The adsorption of one $\mathrm{H}$ on the reconstructed (100) surface is more stabilizing by $0.2 \mathrm{eV}$. This agrees with the findings of Ref. [21]. For both systems a localized spin is observed at C2 
Table 3: Formation energies $\left(\mathrm{E}_{\text {form }}\right.$ in $\left.\mathrm{eV}\right)$ of the reconstructed surfaces and bilayers per edge carbon and of the mono- $(\operatorname{Vac}(\alpha), \operatorname{Vac}(\beta))$ and 5-8-5-divacancies (Divac) on the (001) surface. $(100)_{r e c} /(110)_{r e c}$ denote the in-plane surface reconstructions; $(100)_{\text {arch }} /(110)_{\text {arch }}$ and $(100)_{\text {arch }}^{\text {bilayer }} /(110)_{\text {arch }}^{\text {bilayer }}$ denote the slab and bilayer models of the arch-shaped reconstruction, respectively.

\begin{tabular}{cc}
\hline System & $\mathrm{E}_{\text {form }}$ \\
\hline$(100)_{\text {rec }}$ & -0.55 \\
$(110)_{\text {rec }}$ & 0.15 \\
$(100)_{\text {arch }}$ & -1.61 \\
$(110)_{\text {arch }}$ & -1.04 \\
$(100)_{\text {archer }}^{\text {arch }}$ & -2.04 \\
$(110)_{\text {birch }}^{\text {biayer }}$ & -1.37 \\
$\operatorname{Vac}(\alpha)$ & 7.39 \\
$\operatorname{Vac}(\beta)$ & 7.35 \\
$\operatorname{Divac}$ & 6.94 \\
\hline
\end{tabular}

(the $\mathrm{C}-\mathrm{H}$ bond is formed with $\mathrm{C} 1$ ), the ground state has a magnetic moment of $1.0 \mu_{B}$. The $\mathrm{H}$ adsorption locally deforms the surface $\left(\mathrm{E}_{\text {def }}\right.$ is $0.93 \mathrm{eV}$ and $0.79 \mathrm{eV}$ for the (100) and (110) surface, respectively).

The second $\mathrm{H}$ can again be adsorbed in two different ways same as for the (110) surface as shown in Figure 4. These structures are called $100_{\text {rec}^{-}}$ $2 \mathrm{H}-\mathrm{a} / 110_{r e c}-2 \mathrm{H}-\mathrm{a}$ and $100_{r e c}-2 \mathrm{H}-\mathrm{b} / 110_{r e c}-2 \mathrm{H}-\mathrm{b} .100_{r e c}-2 \mathrm{H}-\mathrm{a} / 110_{r e c}-2 \mathrm{H}-\mathrm{a}$ are the more stable configurations since the deformation is much less pronounced ( $\mathrm{E}_{\text {def }}$ is $0.3-0.4 \mathrm{eV}$ smaller) and the ground state is non-magnetic with no localized spins. Configurations $100_{\text {rec }}-2 \mathrm{H}-\mathrm{b}$ and $110_{\text {rec }}-2 \mathrm{H}-\mathrm{b}$ are less stable, $\mathrm{E}_{a d}$ per $\mathrm{H}$ atom differs by $1.0 \mathrm{eV}$ and $1.1 \mathrm{eV}$, respectively. For these structures a second localized spin is found at $\mathrm{C} 4$ (the second $\mathrm{C}-\mathrm{H}$ bond is formed with C3), however the ground state is also non-magnetic. $\mathrm{E}_{\text {def }}$ is about two times higher than for adsorption of one $\mathrm{H}$. Thus for $\mathrm{H}$ adsorption, the same tendencies as for the (110) surface are found.

For the arch-shaped reconstruction the curvature leads to an increased $s p^{3}$ character of the surface carbon atoms even though no dangling bonds remain. A measure of this curvature can be the dihedral angle between an edge carbon and its three next neighbors. The angles are $39^{\circ}$ and $40^{\circ}$ for the (100) and (110) slab models, and $17^{\circ}$ and $18^{\circ}$ for the (100) and (110) bilayer models, respectively (the angles for the (001) surface of graphite and diamond are $0^{\circ}$ and $60^{\circ}$, respectively). Another measure is the maximum separation between the two layers. It is 4.1 and $4.0 \AA$ for the (100) and (110) slab models, respectively and $9.0 \AA$ for both bilayer models. Both of these measures show that the curvature is much higher for the slab models. 


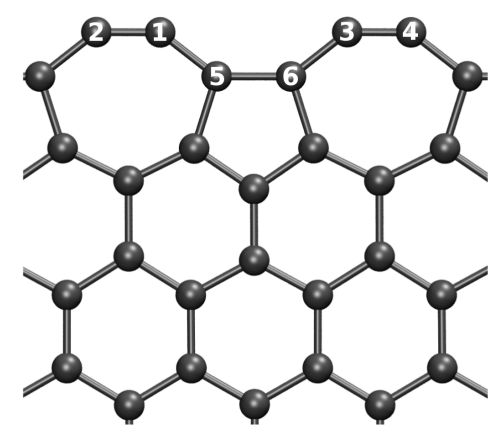

(a)
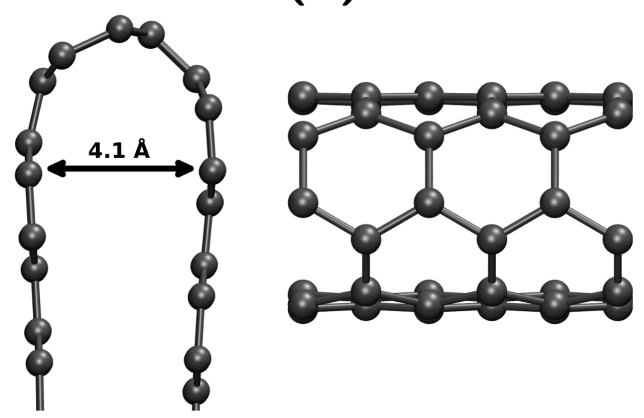

(c)
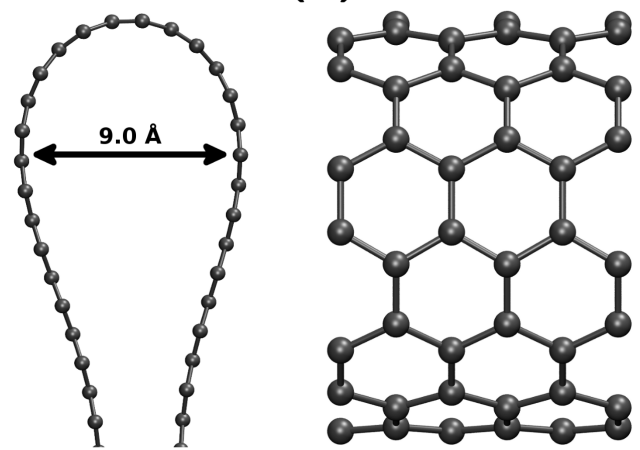

(e)

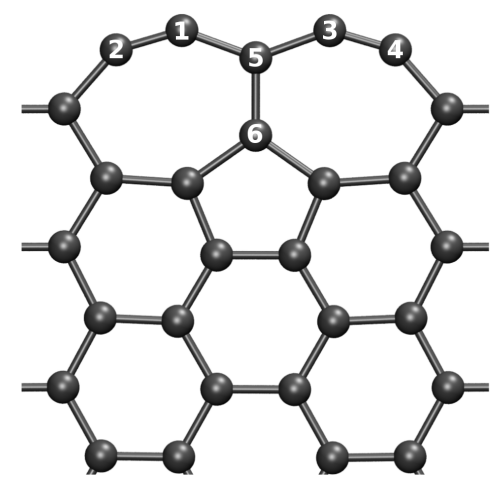

(b)
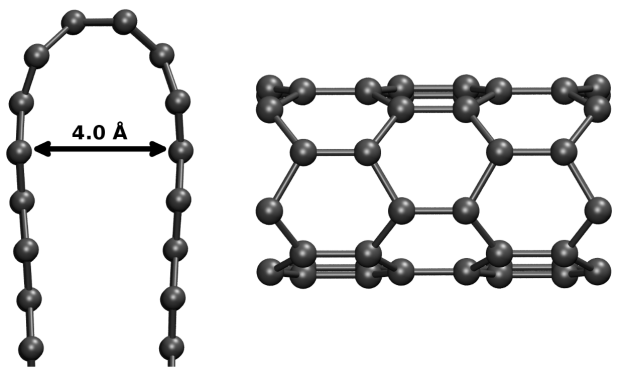

(d)
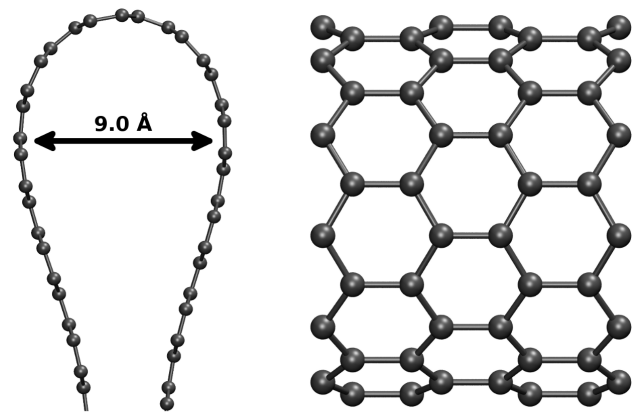

(f)

Figure 3: Reconstructed surfaces of in-plane and arch-shaped reconstructions. In-plane reconstruction of the (a) (100) and (b) (110) surface, arch-shaped reconstructions for (c) (100) and (d) (110) slab models, and for (e) (100) and (f) (110) bilayer models. For (c)(f) the left and right pictures are their side and top views (perpendicular to the z-axis), respectively. Slab models are periodic in $\mathrm{x}$ - and $\mathrm{y}$-directions, bilayer models only in the $\mathrm{x}$-direction.

A higher curvature increases the the $\mathrm{C}-\mathrm{H}$ bond strength; thus adsorption of atomic $\mathrm{H}$ is more stable by a factor of 2 for the bilayer models and 4 for the 
slab models compared to the (001) surface. This trend agrees qualitatively with the findings of Ruffieux et al. [88], who compared hydrogen adsorption on graphite to a carbon nanotube and $\mathrm{C}_{60}$, as well as Tozzini et al. [89], who studied hydrogen adsorption on curved graphene. Both found an increasing $\mathrm{C}-\mathrm{H}$ bond strength with increasing curvature. This leads to a shortening of the $\mathrm{C}-\mathrm{H}$ bond to about $1.10 \AA$ compared to $1.13 \AA$ on the $(001)$ surface. As is also observed for the pristine (001) surface the formation of the $\mathrm{C}-\mathrm{H}$ bond lifts the $\mathrm{C}$ atom by about 0.25 and $0.18 \AA$ for the reconstructed (100) and (110) surfaces, respectively. $\mathrm{E}_{a d}$ for both surfaces is almost the same, which is due to their similar curvature.

The $\mathrm{H}$ atoms have the tendency to form dimers [35] and clusters [90] on the graphene planes. For the adsorption of a second $\mathrm{H}$ atom on the (001) surface it was shown that the preferred adsorption sites are in vicinity of the first hydrogen [34]. For the ortho and para configurations, adsorption of two hydrogen atoms is a more stabilizing process (by a factor of two) than the adsorption of two isolated hydrogen atoms. This leads to the preferred formation of dimers [35] and clustering [90]. The order of stability is ortho $>$ para $>$ meta for these three configurations [34]. However, the ortho and para configuration differ only by $0.03 \mathrm{eV}$.

For the reconstructed (110) slab model the order is switched to para > ortho $>$ meta, but $\mathrm{E}_{a d}$ of all three configurations lies in a close range of 0.2 $\mathrm{eV}$ (as opposed to $1.1 \mathrm{eV}$ for the (001) surface[34]). For the reconstructed (100) slab we find the same order for the two configurations studied, i.e. ortho $>$ meta (the para configuration was omitted since the $\mathrm{C}$ atoms in para position are at least $0.4 \AA$ below the surface).

For our bilayer models the trend of Ref. [34] is reproduced. Compared to the slab models, $\mathrm{H}$ adsorption is a less stabilizing process due to the smaller initial curvature as well as the higher flexibility of the bilayers which leads to a higher deformation. The maximum separation between the two layers decreases by $1.3 \AA$ for one hydrogen and by $1.9-2.7 \AA$ for two chemisorbed hydrogen atoms. This leads to $\mathrm{E}_{\text {def }}$, which are 50-100\% higher compared to the slab models (see Table 2). Figure 4 shows the optimized structure for adsorption of hydrogen on the slab model surfaces (the ones for the bilayer model surfaces are shown in the Supporting Information).

It should be noted that $\mathrm{E}_{a d}$ more than doubles (which means $\mathrm{E}_{a d}$ per $\mathrm{H}$ atom decreases) for all reconstructed surface models when a second $\mathrm{H}$ adsorbs in either the ortho, meta, or para position. Thus, we also find a preferred formation of dimers same as for the pristine (001) surface. 


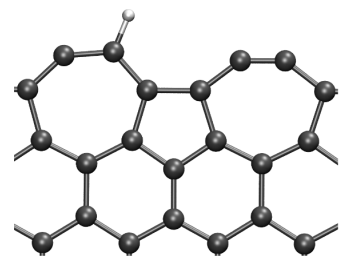

(a)

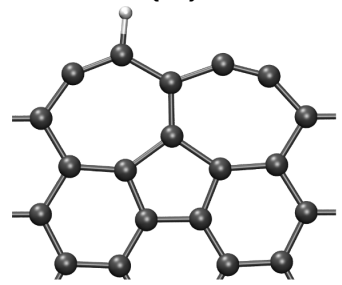

(d)

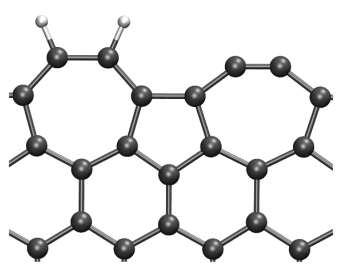

(b)

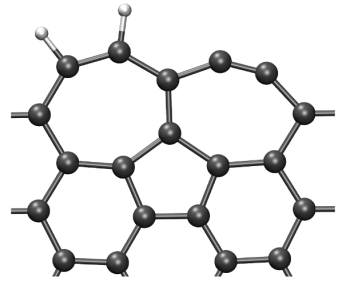

(e)

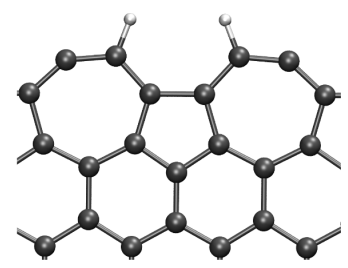

(c)

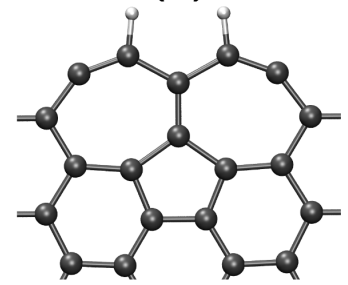

(f)

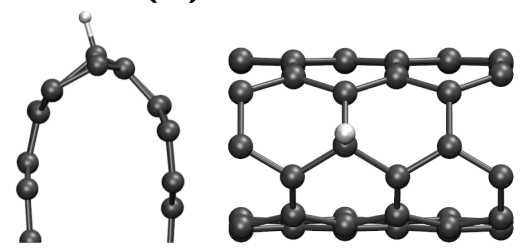

(g)
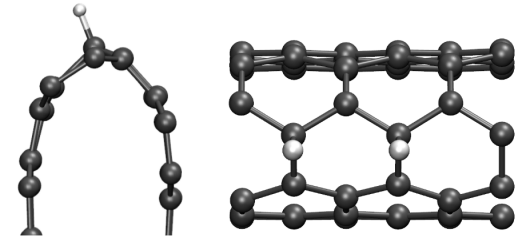

(i)
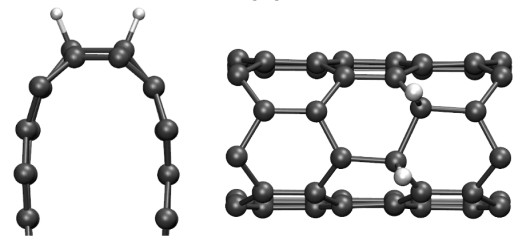

(k)
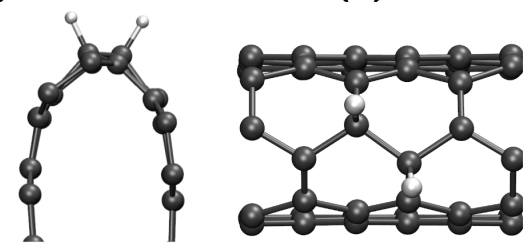

(h)
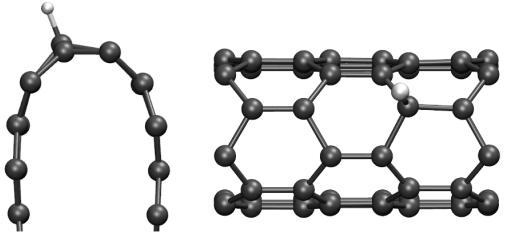

(j)
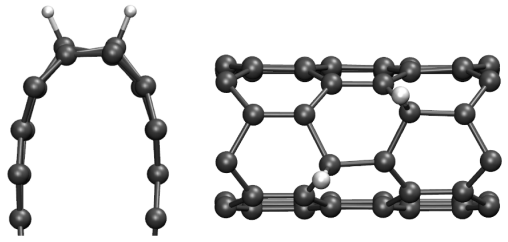

(I)

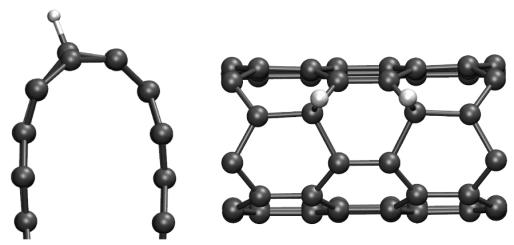

(m)

Figure 4: Optimized structures of different cases of hydrogen adsorption on the reconstructed (100) and (110) surfaces: The in-plane reconstructed (100) surface with (a) one $\mathrm{H}$ and two $\mathrm{H}$ for (b) configuration (100) rec $-2 \mathrm{H}$-a and (c) configuration (100) rec $-2 \mathrm{H}-\mathrm{b}$. The in-plane reconstructed (110) surface with (d) one $\mathrm{H}$ and two $\mathrm{H}$ for (e) configuration $(110)_{\text {rec }}-2 \mathrm{H}-\mathrm{a}$ and (f) configuration (110) req-2H-b. The arch-shaped reconstructed (100) surface with (g) one $\mathrm{H}$ and two $\mathrm{H}$ in (h) ortho and (i) meta positions; the reconstructed (110) surface with $(\mathrm{j})$ one $\mathrm{H}$, two $\mathrm{H}$ in $(\mathrm{k})$ ortho, (l) meta and $(\mathrm{m})$ para positions. The left and right pictures for $(\mathrm{g})-(\mathrm{m})$ are their side and top views, respectively. 
Atsumi reports an adsorption enthalpy of $-2.6 \mathrm{eV} / \mathrm{H}_{2}$ for $\mathrm{H}_{2}$ adsorption on graphite [11] in agreement with the extrapolated adsorption enthalpy of $-2.5 \mathrm{eV} / \mathrm{H}_{2}$ obtained by Redmond and Walker [91] . For the studied in-plane reconstructions, the adsorption energies with respect to $\mathrm{H}_{2}$ are $-3.1 \mathrm{eV} / \mathrm{H}_{2}$ for configuration $(100)_{\text {rec }}-2 \mathrm{H}-\mathrm{a}$ and $-2.9 \mathrm{eV} / \mathrm{H}_{2}$ for $(110)_{\text {rec }}-2 \mathrm{H}-\mathrm{a}$. For the archshaped reconstructions, the lowest adsorption energies with respect to $\mathrm{H}_{2}$ are -1.2 and $-0.8 \mathrm{eV}$ for the (100) and (110) slab, respectively. For both types of reconstructions these results agree with Kanashenko's hypothesis that the (100) edges of crystallites are preferred. However, the differences are rather small which implies that both reconstructed surfaces can be traps. Compared to experiment the in-plane reconstructions underestimate and arch-shaped reconstructions overestimate the adsorption energy. However, the in-plane reconstructions are in better agreement with experiment which suggests that this type is predominantly found in nuclear graphite. Finally, taking into account that the in-plane reconstruction of the (110) surface is endothermic, this suggests that the second trap is indeed the in-plane reconstructed (100) surface of crystallites.

In the next section we will present the influence of point defects, which for instance are created by irradiation, on the adsorption properties of hydrogen.

\subsection{Point defects}

Creation of monovacancies and divacancies at the (001) surface by removal of a carbon atom is a highly endothermic process. These defects were already studied in detail by several authors [46, 48, 49]. The three treated vacancies are shown in Figure 5. Our $\mathrm{E}_{\text {form }}$ for the creation of a monovacancy (7.4 eV, see Table 3) agrees well with literature values of $7.5 \pm 0.5$ $\mathrm{eV}$ (see Ref. [1] and references therein). A monovacancy leads to a surface reconstruction with one dangling bond remaining. The formed $\mathrm{C} 1-\mathrm{C} 2$ bond is rather weak with a bond length of $2.05 \AA$. Due to the dangling bond the local spin density on the unsaturated carbon is $\approx 0.97 \mu_{B}$ (the total magnetic moment is about $\left.1.5 \mu_{B}\right)$. For graphite two different monovacancies are possible. For the $\alpha$-vacancy the vacancy is atop of a carbon atom of the graphene plane underneath the surface plane, whereas for the $\beta$-vacancy it is atop the center of a six-membered ring of the underlying plane.

For divacancies several reconstruction processes are known [92]. For the 5-8-5 divacancy the surface reconstructs in a manner that no dangling bond remains and the ground state is non-magnetic. This reconstruction is also found for extended vacancy lines [93], which shows that the model system is a reasonable approximation to bigger systems.

In Table 2 the adsorption energies for the saturation of monovacancies with atomic hydrogen are shown. 


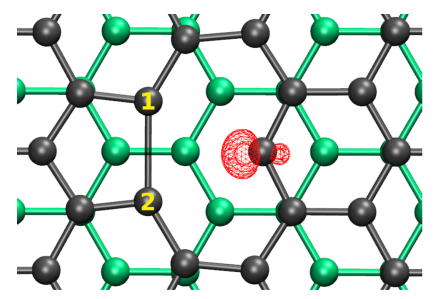

(a)

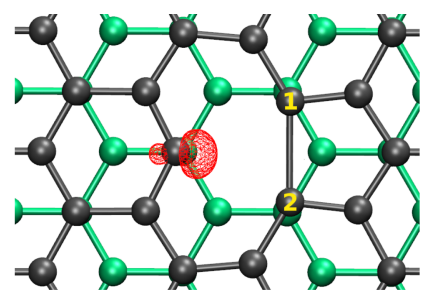

(b)

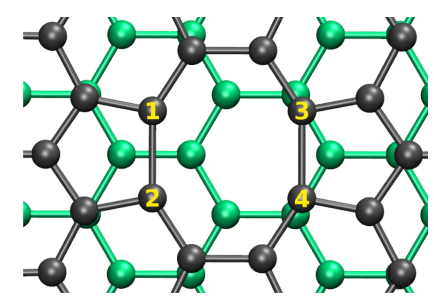

(c)

Figure 5: Top view of the optimized structures for the (a) $\alpha$ - and (b) $\beta$-vacancies and (c) the 5-8-5 divacancy at the (001) surface. Isovalues for spin density plots are $0.05 \mathrm{e} / \mathrm{bohr}^{3}$.

The saturation of the dangling bond leads to a non-magnetic ground state. As can be seen in Figure 6 hydrogen does not remain in the graphene plane when it is chemisorbed on the vacancy on the (001) surface. Two conformations with very similar adsorption energies were found. The first and more stable one is similar to the findings of Allouche et al. [50], where the hydrogen and its bonded carbon are above the graphene plane. For the second conformation both are directed toward the plane lying below the surface. $\Delta \mathrm{E}$ between the two conformations is only $0.02 \mathrm{eV}$. Our adsorption energies ( -4.15 and $-4.17 \mathrm{eV}$ for the $\alpha$ and $\beta$ vacancy, respectively) are in good agreement with the published results for adsorption at a vacancy in graphene (-4.36 eV [49] and $-4.14 \mathrm{eV}[50])$.

A second hydrogen can be adsorbed on the carbon where the first hydrogen is adsorbed to. $\mathrm{E}_{a d}$ for this process is $1.7 \mathrm{eV}$ less than twice $\mathrm{E}_{a d}$ for the first hydrogen due to the $s p^{3}$ hybridization and larger deformation of the lattice. Again, we find two different conformations for both vacancies which are similar in stability $(\Delta \mathrm{E}=0.04 \mathrm{eV})$. For the first one both hydrogen atoms as well as the carbon are above the graphene plane. For the second one the carbon is lying in the graphene plane, one hydrogen is above and one below the surface. We do not find a stable conformation where both hydrogen atoms are beneath the surface. When the bond energy of $\mathrm{H}_{2}$ is taken into account the adsorption energies are about $-2.1 \mathrm{eV}$, thus a dissociation and adsorption of $\mathrm{H}_{2}$ at a monovacancy is a highly exothermic process (even though there is likely a barrier to overcome). Lehtinen et al. [49] and Allouche et al. [50] showed that a second more stable structure exists. Here, one $\mathrm{H}$ atom binds to the unsaturated $\mathrm{C}$ and the second one forms a bond with either $\mathrm{C} 1$ or $\mathrm{C} 2$ (see Figure 5 for the description of atoms).

For the divacancy, there are no dangling bonds; thus the adsorption of hydrogen is much less stable (by $45 \%$ ) compared to vacancies $\alpha$ or $\beta$ (see Table 2). However, the strain in the formed 5-8-5 ring still leads to a stronger $\mathrm{H}$ adsorption than for a pristine (001) surface. The preferred adsorption 


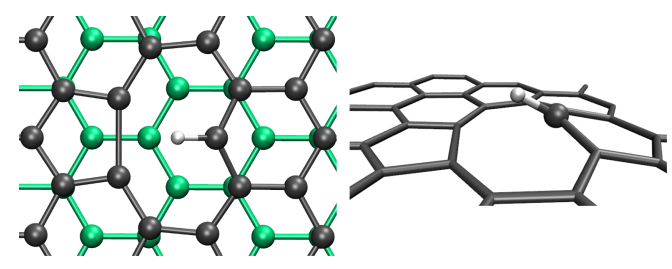

(a)

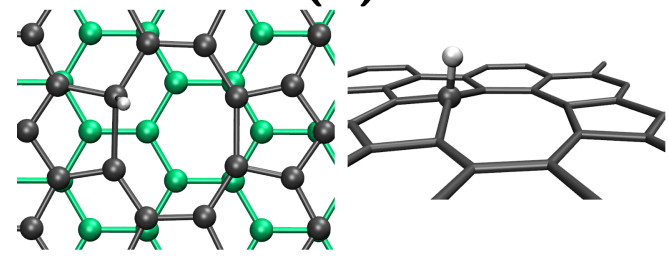

(c)

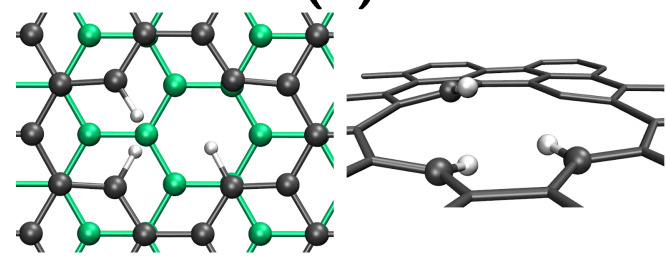

(e)

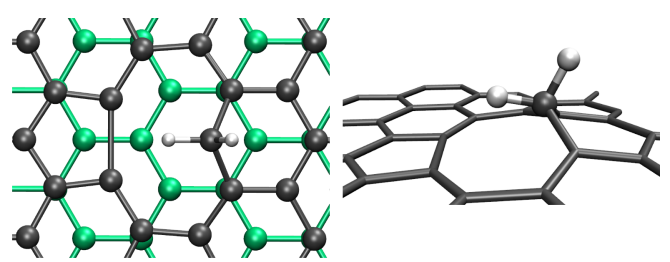

(b)

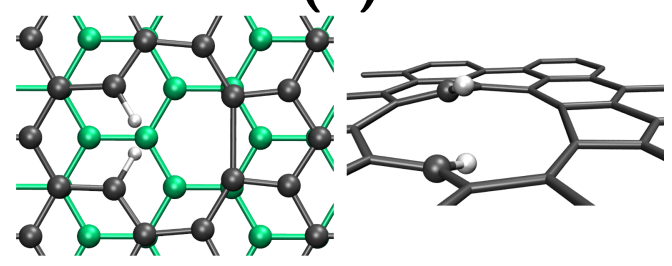

(d)

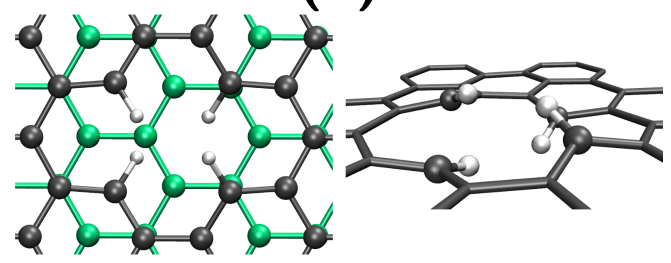

(f)

Figure 6: Top (left) and perspective (right) views of the optimized structures of hydrogen chemisorption on the mono and divacancies. $\operatorname{Vacancy}(\alpha)$ with (a) one and (b) two hydrogen atoms. Divacancy with (c) one, (d) two, (e) three and (f) four hydrogen atoms, respectively.

position is above the carbon (C1) with which the covalent bond is formed (see Figure 6). The elongated bonds make it easier for the carbon to move out of the graphene plane by about $0.15 \AA$. However, the relative rigidity of the graphene plane leads to a structure, which resembles the adsorption on the pristine (001) surface. This results in a $\mathrm{C}-\mathrm{H}$ bond length of $1.11 \AA$, thus $0.03 \AA$ longer than the usual C-H bond length $(\approx 1.08 \AA)$, but $0.02 \AA$ shorter than for adsorption on the (001) surface.

A second hydrogen can either adsorb on $\mathrm{C} 2$ or on the opposite end of the divacancy $(\mathrm{C} 3 / \mathrm{C} 4)$. Here, we only consider the first case since it is thermodynamically more stable ( $\mathrm{E}_{a d}$ for the second case is about twice the $\mathrm{E}_{a d}$ for one chemisorbed hydrogen). For this configuration one hydrogen is above and one below the surface since this minimizes steric repulsion between the hydrogen atoms. The C1-C2 bond is broken by this process. Even though this bond breaking costs energy ( $\mathrm{E}_{\text {def }}$ increases by $1.4 \mathrm{eV}$ ), $\mathrm{E}_{a d}$ is much more (1.0 eV per $\mathrm{H}$ atom) than twice the $\mathrm{E}_{a d}$ for the first hydrogen. The reason for this large stabilization is the increased strength of the $\mathrm{C}-\mathrm{H}$ bond, which 
shortens the $\mathrm{C}-\mathrm{H}$ bonds to $1.08 \AA$. For this configuration, $\mathrm{E}_{a d}$ with respect to $\mathrm{H}_{2}$ is also $-2.1 \mathrm{eV}$, thus dissociation at a divacancy also is exothermic (although this dissociation also likely has a barrier).

To fully saturate the divacancy two hydrogen atoms can adsorb on the opposite side of the divacancy. The adsorption of a third $\mathrm{H}$ does not resemble the first process. $\mathrm{E}_{a d}$ per $\mathrm{H}$ atom is $1.0 \mathrm{eV}$ more stabilizing compared to the first $\mathrm{H}$ even though $\mathrm{E}_{\text {def }}$ of the lattice is $0.6 \mathrm{eV}$ higher (when comparing the difference between $\mathrm{E}_{\text {def }}$ for 3 and $2 \mathrm{H}$ to that for one $\mathrm{H}$ ). For the relaxed geometry the $\mathrm{H}$ lies in the graphene plane. Contrary to the first adsorption, the $\mathrm{C} 3-\mathrm{C} 4$ bond is very elongated in the initial state due to the strain induced by the two adsorbed $\mathrm{H}$. The bond length is $2.08 \AA$ but no spin localization is observed, which implies a very weak bond that can easily be broken by $\mathrm{H}$ adsorption. A localized spin can be observed on $\mathrm{C} 4$, when $\mathrm{H}$ forms a bond with C3. Finally, for a full saturation of this defect the most stable conformation has two $\mathrm{H}$ lying above the surface and two beneath the surface: $\mathrm{E}_{a d}$ per $\mathrm{H}$ atom is the most stable for four hydrogen atoms. The increase of only $0.3 \mathrm{eV}$ for $\mathrm{E}_{\text {def }}$ shows that $\mathrm{C} 4$ in the initial state is already wellpositioned for the adsorption.

Hydrogen binds to vacancies and divacancies in graphite bulk as strongly as on the (001) surface. The trapped hydrogen atoms are located in the interlayer space. However, since the formation energies are slightly lower $(\approx$ $0.05 \mathrm{eV}$ ) than $\mathrm{E}_{a d}$ on the surface, this shows that steric repulsion is negligible when hydrogen is trapped at a vacancy in the bulk. This correlates well with the change of the lattice parameter $c$, which only increases by 0.02 to $0.06 \AA$. It should be noted that the only stable conformation for two hydrogen atoms at a monovacancy in bulk graphite is the one with one hydrogen above and one below the graphene plane. This explains the lower $\mathrm{E}_{\text {def }}$ since the lattice is hardly perturbed.

To summarize, point defects caused by irradiation also serve as a strong trap for hydrogen. Extended defects, such as the 5-8-5 divacancy, are a weaker trap compared to monovacancies. This is true for the surface as well as for bulk graphite.

\section{Conclusion}

In this paper, we have investigated the influence of surface reconstruction and point defects on the adsorption properties of hydrogen for nuclear graphite. Several models of defects and surfaces have been studied and compared with the supposed traps, i.e. the zig zag edge of dislocation loops and reconstructed surfaces of graphite crystallites. The influence of local and extended defects due to irradiation damage has also been explored. 
For adsorption on the surfaces (001), (100), and (110) the obtained relative stabilities are $(100)>(110)>(001)$. Thus, the (100) surface forms the most stable structures with hydrogen. This is directly related to the surface energies of these three surfaces. The most stable structures always possess one $\mathrm{C}-\mathrm{H}$ bond per edge carbon. We have checked the hypothesis of trap 1 in nuclear graphite (zigzag edges of dislocation loops) and found a good agreement between our $\mathrm{E}_{a d}$ on the (100) and Kanashenko's estimation after correcting an error in Kanashenko's assumption of the $\mathrm{C}-\mathrm{H}$ bond energy. However, compared to the experiment, our adsorption energies are overestimated for the (100) surface and underestimated for the (110) surface. We attribute this mismatch to the difficulty to compare experimental data of complex ill defined microstructures measured at elevated temperatures $(>$ $750{ }^{\circ} \mathrm{C}$ ) with theoretical $a b$ initio results of well defined structures at $0 \mathrm{~K}$. Nevertheless, we can conclude that unreconstructed zigzag edges cannot be the only traps in graphite crystallites contrary to Kanashenko's and Atsumi's claims, thus the current hypothesis for the first trap needs to be refined.

Two types of reconstructions of (100) and (110) surfaces have been studied. First, in-plane reconstructions which yield heptagon edge carbon atoms and second, arch-shaped reconstructions which result in no dangling bonds for both systems. For both types we find a decreased stability of $\mathrm{H}$ adsorption compared to unreconstructed surfaces. For arch-shaped reconstructions hydrogen adsorption is considerably stronger than on a pristine (001) surface due to the curvature of the surface. Hydrogen adsorbs stronger on the reconstructed (100) surfaces which agrees with Kanashenko's hypothesis of the second trap in nuclear graphite. $\mathrm{H}$ adsorption energies on in-plane reconstructed surfaces are in better agreement with experiment. Since the in-plane reconstruction of the (110) surface is endothermic, this suggests that the second trap is indeed the in-plane reconstructed (100) surface of crystallites.

Point defects caused by irradiation, such as mono- and divacancies, are also able to bind hydrogen strongly; a divacancy binds hydrogen less strongly than a monovacancy. More extended defects are then weaker traps than monovacancies.

\section{Acknowledgement}

The authors would like to thank the ANRT (French national association for research and technology) for its financial support within the CIFRE agreement 2014/1036 (industrial convention for training through research). 


\section{References}

[1] R. H. Telling, M. I. Heggie, Radiation defects in graphite, Philosophical Magazine 87 (2007) 4797-4846.

[2] J. Kane, C. Karthik, D. Butt, W. Windes, R. Ubic, Microstructural characterization and pore structure analysis of nuclear graphite, J. Nucl. Mater. 415 (2011) 189 - 197.

[3] B. Mironov, H. Freeman, A. Brown, F. Hage, A. Scott, A. Westwood, J.-P. D. Costa, P. Weisbecker, R. Brydson, Electron irradiation of nuclear graphite studied by transmission electron microscopy and electron energy loss spectroscopy, Carbon 83 (2015) 106-117.

[4] H. Atsumi, S. Tokura, M. Miyake, Absorption and desorption of deuterium on graphite at elevated temperatures, J. Nucl. Mater. 155-157 (1988) 241-245.

[5] H. Atsumi, M. Iseki, T. Shikama, Hydrogen solubility and diffusivity in neutron-irradiated graphite, J. Nucl. Mater. 191-194 (1992) 368-372.

[6] H. Atsumi, M. Iseki, T. Shikama, Hydrogen behavior in carbon-based materials and its neutron irradiation effect, J. Nucl. Mater. 233-237 (1996) 1128-1132.

[7] H. Atsumi, M. Iseki, Hydrogen absorption process into graphite and carbon materials, J. Nucl. Mater. 283-287 (2000) 1053-1056.

[8] H. Atsumi, Hydrogen bulk retention in graphite and kinetics of diffusion, J. Nucl. Mater. 307-311 (2002) 1466-1470.

[9] H. Atsumi, Hydrogen retention in graphite and carbon materials under a fusion reactor environment, J. Nucl. Mater. 313-316 (2003) 543-547.

[10] H. Atsumi, K. Tauchi, Hydrogen absorption and transport in graphite materials, J. Alloy. Compd. 356-357 (2003) 705-709.

[11] H. Atsumi, T. Tanabe, T. Shikama, Hydrogen behavior in carbon and graphite before and after neutron irradiation trapping, diffusion and the simulation of bulk retention, Journal of Nuclear Materials 417 (1) (2011) 633-636.

[12] H. Atsumi, Y. Takemura, T. Miyabe, T. Konishi, T. Tanabe, T. Shikama, Desorption of hydrogen trapped in carbon and graphite, Journal of Nuclear Materials 442 (1) (2013) S746-S750. 
[13] S. Kanashenko, A. Gorodetsky, V. Chernikov, A. Markin, A. Zakharov, B. Doyle, W. Wampler, Hydrogen adsorption on and solubility in graphites, J. Nucl. Mater. 233-237 (1996) 1207-1212.

[14] C. Coulson, M. Poole, Calculations of the formation energy of vacancies in graphite crystals, Carbon 2 (1964) 275-279.

[15] T. Wassmann, A. P. Seitsonen, A. M. Saitta, M. Lazzeri, F. Mauri, Structure, stability, edge states, and aromaticity of graphene ribbons, Phys. Rev. Lett. 101 (2008) 096402.

[16] A. Krasheninnikov, K. Nordlund, Ion and electron irradiation-induced effects in nanostructured materials, J. Appl. Phys. 107 (2010) 071301.

[17] S. Dutta, S. K. Pati, Edge reconstructions induce magnetic and metallic behavior in zigzag graphene nanoribbons, Carbon 48 (2010) 4409-4413.

[18] J. Kunstmann, C. Özdoğan, A. Quandt, H. Fehske, Stability of edge states and edge magnetism in graphene nanoribbons, Phys. Rev. B 83 (2011) 045414.

[19] F. Banhart, J. Kotakoski, A. V. Krasheninnikov, Structural defects in graphene, ACS Nano 5 (2011) 26-41.

[20] R. Nair, M. Sepioni, I.-L. Tsai, O. Lehtinen, J. Keinonen, A. Krasheninnikov, T. Thomson, A. Geim, I. Grigoreiva, Spin-half paramagnetism in graphene induced by point defects, Nature Physics 8 (2012) 199-202.

[21] P. Koskinen, S. Malola, H. Häkkinen, Self-passivating edge reconstructions of graphene, Phys. Rev. Lett. 101 (2008) 115502.

[22] L. Jeloaica, V. Sidis, DFT investigation of the adsorption of atomic hydrogen on a cluster-model graphite surface, Chem. Phys. Lett. 300 (1999) 157-162.

[23] X. Sha, B. Jackson, First-principles study of the structural and energetic properties of H atoms on a graphite (0001) surface, Surface Science 496 (2002) 318-330.

[24] S. Patchkovskii, J. S. Tse, S. N. Yurchenko, L. Zhechkov, T. Heine, G. Seifert, Graphene nanostructures as tunable storage media for molecular hydrogen, Proceedings of the National Academy of Sciences of the United States of America 102 (2005) 10439-10444. 
[25] M. Bonfanti, R. Martinazzo, G. F. Tantardini, A. Ponti, Physisorption and diffusion of hydrogen atoms on graphite from correlated calculations on the hcoronene model system, The Journal of Physical Chemistry C 111 (2007) 5825-5829.

[26] G. M. Psofogiannakis, G. E. Froudakis, DFT study of the hydrogen spillover mechanism on pt-doped graphite, The Journal of Physical Chemistry C 113 (2009) 14908-14915.

[27] R. M. Ferullo, N. F. Domancich, N. J. Castellani, On the performance of van der Waals corrected-density functional theory in describing the atomic hydrogen physisorption on graphite, Chem. Phys. Lett. 500 (2010) 283-286.

[28] M. Rubes, J. Kysilka, P. Nachtigall, O. Bludsky, DFT/CC investigation of physical adsorption on a graphite (0001) surface, Phys. Chem. Chem. Phys. 12 (2010) 6438-6444.

[29] F. Costanzo, P. L. Silvestrelli, F. Ancilotto, Physisorption, diffusion, and chemisorption pathways of $\mathrm{H}_{2}$ molecule on graphene and on $(2,2)$ carbon nanotube by first-principles calculations, J. Chem. Theor. Comp. 8 (2012) 1288-1294.

[30] M. Darvish Ganji, S. M. Hosseini-khah, Z. Amini-tabar, Theoretical insight into hydrogen adsorption onto graphene: A first-principles B3LYPD3 study, Phys. Chem. Chem. Phys. 17 (2015) 2504-2511.

[31] T. Zecho, A. Güttler, X. Sha, B. Jackson, J. Küppers, Adsorption of hydrogen and deuterium atoms on the (0001) graphite surface, J. Chem. Phys. 117 (2002) 8486-8492.

[32] A. Allouche, Y. Ferro, T. Angot, C. Thomas, J.-M. Layet, Hydrogen adsorption on graphite $(0001)$ surface: A combined spectroscopy-densityfunctional-theory study, J. Chem. Phys. 123 (2005) 124701.

[33] D. W. Boukhvalov, M. I. Katsnelson, A. I. Lichtenstein, Hydrogen on graphene: Electronic structure, total energy, structural distortions and magnetism from first-principles calculations, Phys. Rev. B 77 (2008) 035427 .

[34] Ž. Šljivančanin, E. Rauls, L. Hornekær, W. Xu, F. Besenbacher, B. Hammer, Extended atomic hydrogen dimer configurations on the graphite (0001) surface, J. Chem. Phys. 131 (2009) 084706. 
[35] R. Balog, B. Jørgensen, J. Wells, E. Lægsgaard, P. Hofmann, F. Besenbacher, L. Hornekær, Atomic hydrogen adsorbate structures on graphene, J. Am. Chem. Soc. 131 (2009) 8744-8745.

[36] V. V. Ivanovskaya, A. Zobelli, D. Teillet-Billy, N. Rougeau, V. Sidis, P. R. Briddon, Hydrogen adsorption on graphene: A first-principles study, Eur. Phys. J. B 76 (2010) 481-486.

[37] Y. Lu, Y. P. Feng, Adsorptions of hydrogen on graphene and other forms of carbon structures: First-principles calculations, Nanoscale 3 (2011) $2444-2453$.

[38] F. H. Yang, R. T. Yang, Ab initio molecular orbital study of adsorption of atomic hydrogen on graphite: Insight into hydrogen storage in carbon nanotubes, Carbon 40 (2002) 437-444.

[39] W. A. Diño, H. Nakanishi, H. Kasai, T. Sugimoto, T. Kondo, $\mathrm{H}_{2}$ dissociative adsorption at the zigzag edges of graphite, J. Surf. Sci. Nanotechnol. 2 (2004) 77-80.

[40] W. A. Diño, Y. Miura, H. Nakanishi, H. Kasai, T. Sugimoto, T. Kondo, $\mathrm{H}_{2}$ dissociative adsorption at the armchair edges of graphite, Solid State Commun. 132 (2004) 713-718.

[41] X. Sha, B. Jackson, The location of adsorbed hydrogen in graphite nanostructures, J. Am. Chem. Soc. 126 (2004) 13095-13099.

[42] L. Li, S. Reich, J. Robertson, Defect energies of graphite: Densityfunctional calculations, Phys. Rev. B 72 (2005) 184109.

[43] J. Ma, D. Alfè, A. Michaelides, E. Wang, Stone-wales defects in graphene and other planar $s p^{2}$-bonded materials, Phys. Rev. B 80 (2009) 033407.

[44] J. C. Meyer, C. Kisielowski, R. Erni, M. D. Rossell, M. F. Crommie, A. Zettl, Direct imaging of lattice atoms and topological defects in graphene membranes, Nano Letters 8 (2008) 3582-3586.

[45] S. Letardi, M. Celino, F. Cleri, V. Rosato, Atomic hydrogen adsorption on a Stone-Wales defect in graphite, Surface Science 496 (2002) 33-38.

[46] R. H. Telling, C. P. Ewels, A. A. El-Barbary, M. I. Heggie, Wigner defects bridge the graphite gap, Nat. Mater. 2 (2003) 333-337. 
[47] G. Teobaldi, H. Ohnishi, K. Tanimura, A. L. Shluger, The effect of van der waals interactions on the properties of intrinsic defects in graphite, Carbon 48 (2010) 4145-4161.

[48] H. Zhang, M. Zhao, X. Yang, H. Xia, X. Liu, Y. Xia, Diffusion and coalescence of vacancies and interstitials in graphite: A first-principles study, Diamond and Related Materials 19 (2010) 1240-1244.

[49] P. O. Lehtinen, A. S. Foster, Y. Ma, A. V. Krasheninnikov, R. M. Nieminen, Irradiation-induced magnetism in graphite: A density functional study, Phys. Rev. Lett. 93 (2004) 187202.

[50] A. Allouche, Y. Ferro, Dissociative adsorption of small molecules at vacancies on the graphite (0001) surface, Carbon 44 (2006) 3320-3327.

[51] M. Casartelli, S. Casolo, G. F. Tantardini, R. Martinazzo, Structure and stability of hydrogenated carbon atom vacancies in graphene, Carbon 77 (2014) 165-174.

[52] E. Kaxiras, K. C. Pandey, Energetics of defects and diffusion mechanisms in graphite, Phys. Rev. Lett. 61 (1988) 2693-2696.

[53] A. Krasheninnikov, P. Lehtinen, A. Foster, R. Nieminen, Bending the rules: Contrasting vacancy energetics and migration in graphite and carbon nanotubes, Chemical Physics Letters 418 (1) (2006) 132-136.

[54] E. Asari, M. Kitajima, K. G. Nakamura, T. Kawabe, Thermal relaxation of ion-irradiation damage in graphite, Phys. Rev. B 47 (1993) 1114311148 .

[55] G.-D. Lee, C. Z. Wang, E. Yoon, N.-M. Hwang, D.-Y. Kim, K. M. Ho, Diffusion, coalescence, and reconstruction of vacancy defects in graphene layers, Phys. Rev. Lett. 95 (2005) 205501.

[56] X. Yang, H. Xia, X. Qin, W. Li, Y. Dai, X. Liu, M. Zhao, Y. Xia, S. Yan, B. Wang, Correlation between the vacancy defects and ferromagnetism in graphite, Carbon 47 (5) (2009) 1399-1406.

[57] A. Ramos, I. Cameán, A. B. García, Graphitization thermal treatment of carbon nanofibers, Carbon 59 (2013) 2-32.

[58] J. Feng, L. Qi, J. Y. Huang, J. Li, Geometric and electronic structure of graphene bilayer edges, Phys. Rev. B 80 (2009) 165407. 
[59] W.-J. Yin, Y.-E. Xie, L.-M. Liu, Y.-P. Chen, R.-Z. Wang, X.-L. Wei, J.-X. Zhong, L. Lau, Atomic structure and electronic properties of folded graphene nanoribbons: A first-principles study, J. Appl. Phys. 113 (2013) 173506.

[60] R. Dovesi, R. Orlando, B. Civalleri, C. Roetti, V. R. Saunders, C. M. Zicovich-Wilson, CRYSTAL: A computational tool for ab initio study of the electronic properties of crystals, Z. Kristallogr. 220 (2005) 571-573.

[61] R. Dovesi, V. R. Saunders, C. Roetti, R. Orlando, C. M. ZicovichWilson, F. Pascale, B. Civalleri, K. Doll, N. M. Harrison, I. J. Bush, P. D'Arco, M. Llunell, CRYSTAL09 User's Manual, University of Torino, Torino (2009).

[62] S. Grimme, J. Antony, S. Ehrlich, H. Krieg, A consistent and accurate $a b$ initio parametrization of density functional dispersion correction (DFTD) for the 94 elements H-Pu, J. Chem. Phys. 132 (2010) 154104.

[63] C. Lechner, B. Pannier, P. Baranek, N. C. Forero-Martinez, H. Vach, First-principles study of the structural, electronic, dynamic, and mechanical properties of HOPG and diamond: Influence of exchangecorrelation functionals and dispersion interactions, J. Phys. Chem. C 120 (2016) 5083-5100.

[64] J. P. Perdew, K. Burke, M. Ernzerhof, Generalized gradient approximation made simple, Phys. Rev. Lett. (1996) 3865-3868.

[65] Y. Baskin, L. Meyer, Lattice constants of graphite at low temperatures, Phys. Rev. 100 (1955) 544-544.

[66] D. Lide, Handbook of Chemistry and Physics, Vol. $71^{\text {th }}$, CRC Press, Boston, 1990-1991.

[67] L. X. Benedict, N. G. Chopra, M. L. Cohen, A. Zettl, S. G. Louie, V. H. Crespi, Microscopic determination of the interlayer binding energy in graphite, Chem. Phys. Lett. 286 (1998) 490-496.

[68] R. Zacharia, H. Ulbricht, T. Hertel, Interlayer cohesive energy of graphite from thermal desorption of polyaromatic hydrocarbons, Phys. Rev. B 69 (2004) 155406.

[69] R. Dovesi, M. Causà, R. Orlando, C. Roetti, V. R. Saunders, Ab initio approach to molecular crystals: A periodic Hartree-Fock study of crystalline urea, J. Chem. Phys. 92 (1990) 7402-7411. 
[70] M. Catti, A. Pavese, R. Dovesi, V. R. Saunders, Static lattice and electron properties of $\mathrm{MgCO}_{3}$ (magnesite) calculated by ab initio periodic Hartree-Fock methods, Phys. Rev. B 47 (1993) 9189-9198.

[71] A. Lichanot, R. Orlando, G. Mallia, M. Mrawa, R. Dovesi, Voh center in magnesium oxide: an ab initio supercell study, Chem. Phys. Lett. 318 (2000) 240-246.

[72] S. Boys, F. Bernardi, The calculation of small molecular interactions by the differences of separate total energies. Some procedures with reduced errors, Mol. Phys. 19 (1970) 553-566.

[73] H. J. Monkhorst, J. D. Pack, Special points for Brillouin-zone integrations, Phys. Rev. B 13 (1976) 5188-5192.

[74] D. G. Anderson, Iterative procedures for nonlinear integral equations, J. ACM 12 (1965) 547-560.

[75] D. R. Hamann, Semiconductor charge densities with hard-core and softcore pseudopotentials, Phys. Rev. Lett. 42 (1979) 662-665.

[76] N. Ota, N. Gorjizadeh, Y. Kawazoe, Multiple spin state analysis in radical carbon edge and oxygen edge graphene-like molecules, Trans. Magn. Soc. Jpn. 35 (2011) 414-419.

[77] S. Casolo, O. M. Løvvik, R. Martinazzo, G. F. Tantardini, Understanding adsorption of hydrogen atoms on graphene, J. Chem. Phys. 130 (2009) 054704.

[78] L. Hornekær, Ž. Šljivančanin, W. Xu, R. Otero, E. Rauls, I. Stensgaard, E. Lægsgaard, B. Hammer, F. Besenbacher, Metastable structures and recombination pathways for atomic hydrogen on the graphite (0001) surface, Phys. Rev. Lett. 96 (2006) 156104.

[79] J. Kerwin, B. Jackson, The sticking of H and D atoms on a graphite (0001) surface: The effects of coverage and energy dissipation, J. Chem. Phys. 128 (2008) 084702.

[80] Y. Ferro, F. Marinelli, A. Allouche, Density functional theory investigation of the diffusion and recombination of $\mathrm{H}$ on a graphite surface, Chem. Phys. Lett. 368 (2003) 609-615.

[81] E. J. Duplock, M. Scheffler, P. J. D. Lindan, Hallmark of perfect graphene, Phys. Rev. Lett. 92 (2004) 225502. 
[82] T. Roman, W. A. Diño, H. Nakanishi, H. Kasai, T. Sugimoto, K. Tange, Hydrogen pairing on graphene, Carbon 45 (2007) 218-220.

[83] L. Chen, A. C. Cooper, G. P. Pez, H. Cheng, Mechanistic study on hydrogen spillover onto graphitic carbon materials, J. Phys. Chem. C 111 (2007) 18995-19000.

[84] G. E. Davico, V. M. Bierbaum, C. H. DePuy, G. B. Ellison, R. R. Squires, The C-H bond energy of benzene, J. Am. Chem. Soc. 117 (1995) 2590-2599.

[85] I. Suarez-Martinez, G. Savini, G. Haffenden, J.-M. Campanera, M. I. Heggie, Dislocations of burgers vector c/2 in graphite, Phys. Status Solidi C 4 (2007) 2958-2962.

[86] S. V. Rotkin, Y. Gogotsi, Analysis of non-planar graphitic structures: from arched edge planes of graphite crystals to nanotubes, Mater. Res. Innov. 5 (2002) 191-200.

[87] J.-J. Adjizian, C. Latham, S. Oberg, P. Briddon, M. Heggie, DFT study of the chemistry of sulfur in graphite, including interactions with defects, edges and folds, Carbon 62 (2013) 256-262.

[88] P. Ruffieux, O. Gröning, M. Bielmann, P. Mauron, L. Schlapbach, P. Gröning, Hydrogen adsorption on $s p^{2}$-bonded carbon: Influence of the local curvature, Phys. Rev. B 66 (2002) 245416.

[89] V. Tozzini, V. Pellegrini, Reversible hydrogen storage by controlled buckling of graphene layers, The Journal of Physical Chemistry C 115 (2011) 25523-25528.

[90] L. Hornekær, E. Rauls, W. Xu, Ž. Šljivančanin, R. Otero, I. Stensgaard, E. Lægsgaard, B. Hammer, F. Besenbacher, Clustering of chemisorbed $\mathrm{H}(\mathrm{D})$ atoms on the graphite (0001) surface due to preferential sticking, Phys. Rev. Lett. 97 (2006) 186102.

[91] J. P. Redmond, P. L. Walker, Hydrogen sorption on graphite at elevated temperatures, J. Chem. Phys. 64 (9) (1960) 1093-1099.

[92] Y. Kim, J. Ihm, E. Yoon, G.-D. Lee, Dynamics and stability of divacancy defects in graphene, Phys. Rev. B 84 (2011) 075445.

[93] T. Trevethan, C. D. Latham, M. I. Heggie, P. R. Briddon, M. J. Rayson, Vacancy diffusion and coalescence in graphene directed by defect strain fields, Nanoscale 6 (2014) 2978-2986. 